\title{
Functional Inequalities for Two-Level Concentration
}

\author{
Franck Barthe ${ }^{1} \cdot$ Michał Strzelecki $^{2}$
}

Received: 3 October 2019 / Accepted: 7 January 2021 / Published online: 3 July 2021

(C) The Author(s) 2021

\begin{abstract}
Probability measures satisfying a Poincaré inequality are known to enjoy a dimension-free concentration inequality with exponential rate. A celebrated result of Bobkov and Ledoux shows that a Poincaré inequality automatically implies a modified logarithmic Sobolev inequality. As a consequence the Poincaré inequality ensures a stronger dimension-free concentration property, known as two-level concentration. We show that a similar phenomenon occurs for the Latała-Oleszkiewicz inequalities, which were devised to uncover dimensionfree concentration with rate between exponential and Gaussian. Motivated by the search for counterexamples to related questions, we also develop analytic techniques to study functional inequalities for probability measures on the line with wild potentials.
\end{abstract}

Keywords Beckner-type inequalities · Concentration of measure ·

Modified log-Sobolev inequalities

Mathematics Subject Classification (2010) Primary: 60E15 · Secondary: 26D10

\section{Introduction}

This article is a contribution to the functional approach to concentration inequalities, see, e.g., [19]. We work in the setting of Euclidean spaces $\left(\mathbb{R}^{d},\langle\cdot, \cdot\rangle,|\cdot|\right)$, although most of the results extend to more general settings as Riemannian manifolds.

Research partially supported by the National Science Centre, Poland, grants no. 2015/19/N/ST1/00891 (MS) and 2017/24/T/ST1/00323 (doctoral scholarship of MS).

Michał Strzelecki

michalst@mimuw.edu.pl

Franck Barthe

barthe@math.univ-toulouse.fr

1 Institut de Mathématiques de Toulouse (UMR 5219), Université de Toulouse \& CNRS, UPS, 31062 Toulouse Cedex 9, France

2 Institute of Mathematics, University of Warsaw, Banacha 2, 02-097 Warsaw, Poland 
First we recall the main functional inequalities which allow to establish concentration properties. A probability measure $\mu$ on $\mathbb{R}^{d}$ satisfies a logarithmic Sobolev inequality if there is a constant $C_{L S}<\infty$ such that for all smooth functions $f: \mathbb{R}^{d} \rightarrow \mathbb{R}$,

$$
\operatorname{Ent}_{\mu}\left(f^{2}\right) \leq C_{L S} \int_{\mathbb{R}^{d}}|\nabla f|^{2} d \mu
$$

where $\operatorname{Ent}_{\mu}(g)=\int g \log g d \mu-\left(\int g d \mu\right) \log \left(\int g d \mu\right)$ is the entropy of a nonnegative function. It is convenient to denote by $C_{L S}(\mu)$ the smallest possible constant $C_{L S}$ in Eq. 1. Classically the inequality tensorizes, meaning $C_{L S}\left(\mu^{\otimes n}\right)=C_{L S}(\mu)$ for all $n$, and the standard Gaussian measure satisfies a logarithmic Sobolev inequality. Conversely, measures with a log-Sobolev inequality enjoy a dimension-free concentration inequality with Gaussian rate: for all $n \geq 1$ and for all measurable $A \subset \mathbb{R}^{n d}$ with $\mu^{\otimes n}(A) \geq \frac{1}{2}$, it holds for all $t>0$,

$$
\mu^{\otimes n}\left(A+t B_{2}\right) \geq 1-e^{-K \frac{t^{2}}{C_{L S}}},
$$

where $K$ is a numerical constant and $B_{2}=B_{2}^{\text {nd }}$ denotes the Euclidean unit ball (of $\mathbb{R}^{n d}$ ). This concentration property can also be formulated in terms of deviations of functions, as we will mention later.

The other main property in the field is the Poincaré inequality. A probability measure $\mu$ on $\mathbb{R}^{d}$ enjoys a Poincaré inequality if there exists a constant $C_{P}<\infty$ such that for all smooth $f: \mathbb{R}^{d} \rightarrow \mathbb{R}$

$$
\operatorname{Var}_{\mu}(f) \leq C_{P} \int_{\mathbb{R}^{d}}|\nabla f|^{2} d \mu
$$

where $\operatorname{Var}_{\mu}(f)=\int f^{2} d \mu-\left(\int f d \mu\right)^{2}$ is the variance of $f$ with respect to $\mu$. Again $C_{P}(\mu)$ denotes the minimal constant for which the inequality holds. The Poincare inequality tensorizes $\left(C_{P}\left(\mu^{\otimes n}\right)=C_{P}(\mu)\right)$ and ensures dimension-free concentration properties with exponential rate: for all $n$ and all $A \subset \mathbb{R}^{n d}$ with $\mu^{\otimes n}(A) \geq \frac{1}{2}$, and for all $t>0$,

$$
\mu^{\otimes n}\left(A+t B_{2}\right) \geq 1-e^{-K \frac{t}{\sqrt{C_{P}}}},
$$

where $K$ is a numerical constant. The symmetric exponential distribution $d v(t)=e^{-|t|} d t / 2$ on $\mathbb{R}$ satisfies a Poincaré inequality, but not the log-Sobolev inequality. In [23], Talagrand proved a stronger concentration property than the above one: if $v^{\otimes n}(A) \geq \frac{1}{2}$ then for all $t>0$,

$$
v^{\otimes n}\left(A+\sqrt{t} B_{2}+t B_{1}\right) \geq 1-e^{-t / C},
$$

for some universal constant $C$, where $B_{p}=B_{p}^{n}=\left\{x \in \mathbb{R}^{n} ; \sum_{i=1}^{n}\left|x_{i}\right|^{p} \leq 1\right\}$.

Talagrand's two-level concentration inequality (4) is doubly sharp: for $A=(-\infty, 0] \times$ $\mathbb{R}^{n-1}$ it captures the exponential behaviour of coordinates marginals of $v^{\otimes n}$, while for $A=$ $\left\{x ; \sum_{i} x_{i} \leq 0\right\}$ using that $B_{1}^{n} \subset\left\{x \in \mathbb{R}^{n} ; \sum_{i} x_{i} \leq 1\right\}$ and $B_{2}^{n} \subset\left\{x \in \mathbb{R}^{n} ; \sum_{i} x_{i} \leq \sqrt{n}\right\}$, one gets

$$
\nu^{\otimes n}\left(\left\{x \in \mathbb{R}^{n} ; \frac{\sum_{i=1}^{n} x_{i}}{\sqrt{n}} \leq \sqrt{t}+\frac{t}{\sqrt{n}}\right\}\right) \geq 1-e^{-t / C},
$$

which is asymptotically of the right order in $t$ when $n \rightarrow \infty$, according to the Central Limit Theorem. 
Talagrand's two-level concentration phenomenon was incorporated in the functional approach by Bobkov and Ledoux: they introduced a modified log-Sobolev nequality which implies concentration of the type Eq. 4. More importantly, they showed that it is implied by the Poincaré inequality (which means that the concentration consequences of that inequality are stronger than Eq. 3). More precisely,

Theorem 1 (Bobkov-Ledoux [9]) Let $\mu$ be a probability measure on $\mathbb{R}^{d}$, which satisfies a Poincaré inequality with constant $C_{P}$. Then for any $c \in\left(0, C_{P}^{-1 / 2}\right)$, there exists $K\left(c, C_{P}\right)<$ $\infty$ such that for all smooth $f: \mathbb{R}^{d} \rightarrow(0, \infty)$ such that pointwise $\left|\frac{\nabla f}{f}\right| \leq c$,

$$
\operatorname{Ent}_{\mu}\left(f^{2}\right) \leq K\left(c, C_{P}\right) \int_{\mathbb{R}^{d}}|\nabla f|^{2} d \mu .
$$

The Central Limit Theorem and an argument of Talagrand [23] roughly imply that if dimension-free concentration in Euclidean spaces occurs, then the rate of concentration cannot be faster than Gaussian, and the measure should be exponentially integrable. In this sense, Poincaré and log-Sobolev inequalities describe the extreme dimension-free properties. The functional approach to concentration with intermediate rate (between exponential and Gaussian) was developed by Latała and Oleszkiewicz [18]. We say that a probability measure $\mu$ on $\mathbb{R}^{d}$ satisfies the Latała-Oleszkiewicz inequality with parameter $r \in[1,2]$, if there exists a constant $C_{L O(r)}<\infty$ such that for every smooth $f: \mathbb{R}^{d} \rightarrow \mathbb{R}$ one has

$$
\sup _{\theta \in(1,2)} \frac{\int_{\mathbb{R}^{d}} f^{2} d \mu-\left(\int_{\mathbb{R}^{d}}|f|^{\theta} d \mu\right)^{2 / \theta}}{(2-\theta)^{2(1-1 / r)}} \leq C_{L O(r)} \int_{\mathbb{R}^{d}}|\nabla f|^{2} d \mu .
$$

Let us stress here that most of the information is encoded in the speed at which $(2-$ $\theta)^{2(1-1 / r)}$ vanishes as $\theta \rightarrow 2^{-}$(by omitting the supremum on the left-hand side of Eq. 5 and only considering a fixed $\theta \in(1,2)$ one gets a significantly weaker inequality). We sometimes omit the dependence in $r$ in the notation when there is no ambiguity on the value of $r$. For $r=1$ the inequality is equivalent to the Poincare inequality. For $r=2$ and the Gaussian measure, such inequalities were first considered by Beckner [7]. Moreover, up to the constants, the inequality for $r=2$ is equivalent to the log-Sobolev inequality (note in particular that the limit as $\theta \rightarrow 2^{-}$of the ratio on the left-hand side is the entropy). Latała and Oleszkiewicz proved that the above functional inequality tensorizes and implies dimension-free concentration with rate $\exp \left(-t^{r}\right)$ : under Eq. 5, if $\mu^{\otimes n}(A) \geq \frac{1}{2}$ then for $t>0$,

$$
\mu^{\otimes n}\left(A+t B_{2}\right) \geq 1-e^{-K\left(t / \sqrt{C_{L O(r)}}\right)^{r}} .
$$

(their proof yields $K=1 / 3$; see also [26] and Section 6 of [6] for an extension to a more general setting). By $\mu_{r}$ we denote the probability measure on the real line with density

$$
d \mu_{r}(t)=\frac{e^{-|t|^{r}} d t}{2 \Gamma(1+1 / r)}, \quad t \in \mathbb{R} .
$$

For $r \in(1,2)$, Latała and Oleszkiewicz [18] showed that $\mu_{r}$ satisfies the inequality (5) with a uniformly bounded constant (in this case $d=1$ ). For these measures, one obtains a dimension-free concentration inequality with a rate corresponding to the tails.

Another approach was suggested by Gentil, Guillin, and Miclo [13], which we present now. For $r \in(1,2]$, we say that a probability measure $\mu$ on $\mathbb{R}^{d}$ satisfies the 
modified log-Sobolev inequality with parameter $r$ if there exists a constant $C_{m L S(r)}<\infty$ such that for every smooth function $f: \mathbb{R}^{d} \rightarrow(0, \infty)$ one has

$$
\operatorname{Ent}_{\mu}\left(f^{2}\right) \leq C_{m L S(r)} \int_{\mathbb{R}^{d}} H_{r^{\prime}}\left(\frac{|\nabla f|}{f}\right) f^{2} d \mu,
$$

where $H_{r^{\prime}}(t):=\max \left\{t^{2},|t|^{r^{\prime}}\right\}$ for $t \in \mathbb{R}$ and $r^{\prime} \geq 2$ is the dual exponent of $r$, defined by $\frac{1}{r}+\frac{1}{r^{\prime}}=1$. This is a natural extension of the modified log-Sobolev inequality of Bobkov and Ledoux, see Theorem 1, which appears as the limit case $r=1$. Indeed, when $r \rightarrow 1^{+}$, $r^{\prime} \rightarrow \infty$ and

$$
\lim _{r^{\prime} \rightarrow \infty} H_{r^{\prime}}(t / c)= \begin{cases}t^{2} / c^{2} & \text { if }|t| \leq c \\ \infty & \text { if }|t|>c .\end{cases}
$$

The modified log-Sobolev inequality tensorizes as follows: if $\mu$ satisfies Eq. 6 , then for any positive integer $n$ and every smooth function $f: \mathbb{R}^{d n} \rightarrow(0, \infty)$ one has

$$
\text { Ent }_{\mu^{\otimes n}}\left(f^{2}\right) \leq C_{m L S(r)} \int_{\mathbb{R}^{d n}} \sum_{i=1}^{n} H_{r^{\prime}}\left(\frac{\left|\nabla_{i} f\right|}{f}\right) f^{2} d \mu^{\otimes n},
$$

where $\nabla_{i} f$ denotes the partial gradient with respect to the $i$-th $d$-tuple of coordinates of $\mathbb{R}^{d n}$. It is proved in [13] that for each $r \in(1,2)$ the measure $\mu_{r}$ satisfies a modified logSobolev inequality (6) with parameter $r$. This allows to recover a two-level concentration inequality of Talagrand [24], extending Eq. 4: for $r \in(1,2)$, if $\mu_{r}^{\otimes n}(A) \geq \frac{1}{2}$, then

$$
\mu_{r}^{\otimes n}\left(A+\sqrt{t} B_{2}+t^{\frac{1}{r}} B_{r}\right) \geq 1-e^{-t / C_{r}} .
$$

In view of Theorem 1, it is natural to conjecture, that similarly the Latała-Oleszkiewicz inequality implies the modified log-Sobolev inequality (and therefore improved two-level concentration), cf. Remark 21 in [5].

\section{Main Result and Organization of the Article}

We are ready to state our main result. Let us emphasize that it is not restricted to measures on the real line and that the dimension $d$ does not enter into the dependence of constants.

Theorem 2 Let $r \in(1,2)$. Let $\mu$ be a probability measure on $\mathbb{R}^{d}$ which satisfies the LatałaOleszkiewicz inequality (5) with parameter $r$ and with constant $C_{L O(r)}$. Then $\mu$ satisfies the modified log-Sobolev inequality (6) with parameter $r$ and a constant $C$ depending only on $C_{L O(r)}$ and $r$. More precisely, one can take $C=\alpha(r) \max \left\{C_{L O(r)}, C_{L O(r)}^{1 / r}\right\}$ for some function $\alpha$.

The concentration property can also be formulated in terms of functions. As shown in [18], the Latała-Oleszkiewicz inequality (5) implies that for any integer $n \geq 1$ and every 1-Lipschitz function $f: \mathbb{R}^{d n} \rightarrow \mathbb{R}$, one has

$$
\mu^{\otimes n}\left(\left|f-\int_{\mathbb{R}^{d n}} f d \mu^{\otimes n}\right| \geq t \sqrt{C_{L O(r)}}\right) \leq 2 \exp \left(-K \min \left\{t^{2}, t^{r}\right\}\right) .
$$

The modified log-Sobolev inequality (6) implies via a modification of Herbst's argument a stronger deviation inequality. Therefore our main theorem ensures that the 
Latała-Oleszkiewicz inequality ensures such an improved concentration. This is the content of the next two corollaries, for which some notation is needed. For $x=\left(x_{1}, \ldots, x_{n}\right) \in \mathbb{R}^{d \cdot n}$ and $p \in(1, \infty)$ denote

$$
\|x\|_{p, 2}:=\left(\sum_{i=1}^{n}\left|x_{i}\right|^{p}\right)^{1 / p}
$$

(here $|\cdot|$ stands for the $\ell_{2}$-norm on $\mathbb{R}^{d}$; in the notation we suppress the roles of $d$ and $n$, but they will always be clear from the context). The norm $\|\cdot\|_{2,2}$ is just the $\ell_{2}$-norm on $\mathbb{R}^{d \cdot n}$ and will be also denoted by $|\cdot|$.

Corollary 1 Let $r \in(1,2)$. Let $\mu$ be a probability measure on $\mathbb{R}^{d}$ which satisfies the Latata-Oleszkiewicz inequality (5) with parameter $r$ and with constant $C_{L O}$. Then there exists a constant $C>0$, depending only on $C_{L O}$ and $r$, such that for any positive integer $n$, any smooth $f: \mathbb{R}^{d n} \rightarrow \mathbb{R}$, and all $t>0$,

$$
\begin{aligned}
& \mu^{\otimes n}\left(\left|f-\int_{\mathbb{R}^{d n}} f d \mu^{\otimes n}\right| \geq t\right) \\
& \quad \leq 2 \exp \left(-\frac{1}{2} \min \left\{\frac{t^{2}}{C \sup _{x \in \mathbb{R}^{d n}}|\nabla f(x)|^{2}}, \frac{t^{r}}{C^{r-1} \sup _{x \in \mathbb{R}^{d \cdot n}}\|\nabla f(x)\|_{r^{\prime}, 2}^{r}}\right\}\right) .
\end{aligned}
$$

Using standard smoothing arguments one can also obtain a result for not necessarily smooth functions, expressed in terms of their Lipschitz constants.

Corollary 2 Let $r \in(1,2)$. Let $\mu$ be a probability measure on $\mathbb{R}^{d}$ which satisfies the Latała-Oleszkiewicz inequality (5) with parameter $r$ and constant $C_{L O}$. Then there exists a constant $C>0$, depending only on $C_{L O}$ and $r$, such that for any positive integer $n$ the following holds: if $f: \mathbb{R}^{d n} \rightarrow \mathbb{R}$ satisfies

$$
\begin{aligned}
& |f(x)-f(y)| \leq L_{2}|x-y|, \\
& |f(x)-f(y)| \leq L_{r, 2}\|x-y\|_{r, 2},
\end{aligned}
$$

for all $x, y \in \mathbb{R}^{d \cdot n}$, then for all $t>0$,

$$
\mu^{\otimes n}\left(\left|f-\int_{\mathbb{R}^{d n}} f d \mu^{\otimes n}\right| \geq t\right) \leq 2 \exp \left(-\frac{1}{2} \min \left\{\frac{t^{2}}{C L_{2}^{2}}, \frac{t^{r}}{C^{r-1} L_{r, 2}^{r}}\right\}\right) .
$$

Finally, using Corollary 2, we can express concentration in terms of enlargements of sets. Below $B_{2}^{d n}$ and $B_{r}^{d n}$ stand for the unit balls in the $\ell_{2}$ and $\ell_{r}$-norms on $\mathbb{R}^{d n}$, respectively. Also, let

$$
B_{r, 2}^{n, d}:=\left\{\left(x_{1}, \ldots, x_{n}\right) \in \mathbb{R}^{d \cdot n}:\left(\sum_{i=1}^{n}\left|x_{i}\right|^{r}\right)^{1 / r} \leq 1\right\}
$$

be the unit ball in the norm $\|\cdot\|_{r, 2}$. Observe that for $r \in(1,2)$,

$$
d^{1 / 2-1 / r} B_{r, 2}^{n, d} \subset B_{r}^{d n} \subset B_{r, 2}^{n, d} \subset B_{2}^{d n} \subset n^{1 / r-1 / 2} B_{r, 2}^{n, d} .
$$

Corollary 3 Let $\mu$ be a probability measure on $\mathbb{R}^{d}$ which satisfies the Latata-Oleszkiewicz. inequality (5) with parameter $r$ and constant $C_{L O}$. Then there exists a constant $K>0$, 
depending only on $C_{L O}$ and $r$, such that for any positive integer $n$ and any set $A \subset \mathbb{R}^{d n}$ with $\mu^{\otimes n}(A) \geq 1 / 2$,

$$
\mu^{\otimes n}\left(A+\left\{\left(x_{1}, \ldots, x_{n}\right) \in \mathbb{R}^{d \cdot n}: \sum_{i=1}^{n} \min \left\{\left|x_{i}\right|^{2},\left|x_{i}\right|^{r}\right\} \leq t\right\}\right) \geq 1-e^{-K t} .
$$

In particular,

$$
\mu^{\otimes n}\left(A+\sqrt{t} B_{2}^{d n}+t^{1 / r} B_{r, 2}^{n, d}\right) \geq 1-e^{-K t} .
$$

One can take $K=\frac{1}{32} \min \left\{1 / C, 1 / C^{r-1}\right\}$, where $C$ is taken from Theorem 2.

This corollary should be compared with the results of Gozlan [15]. He proved that if a probability measure $\mu$ on $\mathbb{R}^{d}$ satisfies the Latała-Oleszkiewicz inequality, then it satisfies a Poincaré type inequality involving a non-standard length of the gradient (see Corollary 5.17 in [15]), which in turn implies a slightly different type of two-level concentration (see Proposition 2.4 and Proposition 1.2 in [15]). However, unlike in the above two corollaries, the constants which appear in his formulations do depend on the dimension $d$ of the underlying space (even though they do not depend on $n$ ). Namely, if we denote $x_{i}=\left(x_{i}^{1}, \ldots, x_{i}^{d}\right) \in \mathbb{R}^{d}$ for $i=1, \ldots, n$, then [15] shows the existence of a constant $K>0$ (depending only on $C_{L O}$ and $r$ ) such that for any positive integer $n$ and any set $A \subset \mathbb{R}^{d n}$ with $\mu^{\otimes n}(A) \geq 1 / 2$,

$$
\mu^{\otimes n}\left(A+\left\{\left(x_{1}, \ldots, x_{n}\right) \in \mathbb{R}^{d \cdot n}: \sum_{i=1}^{n} \sum_{j=1}^{d} \min \left\{\left|\frac{x_{i}^{j}}{d}\right|^{2},\left|\frac{x_{i}^{j}}{d}\right|^{r}\right\} \leq t\right\}\right) \geq 1-e^{-K t / d}
$$

(the $d$ in the denominator on the left-hand side comes from Corollary 5.17 of [15] and the $d$ on the right-hand side-from Proposition 2.4 therein). In particular, this implies

$$
\mu^{\otimes n}\left(A+d^{3 / 2} \sqrt{t} B_{2}^{d n}+d^{1+1 / r} t^{1 / r} B_{r}^{d n}\right) \geq 1-e^{-K t} .
$$

In terms of the dependence on $d$ this is weaker than Eq. 8, since

$$
B_{r, 2}^{n, d} \subset d^{1 / r-1 / 2} B_{r}^{d n} \subset d^{1+1 / r} B_{r}^{d n},
$$

with strict inclusions when $d \geq 2$.

The organization of the rest of the article is the following. In Section 3 we introduce two more functional inequalities. They will serve as intermediate steps between the LatałaOleszkiewicz and the modified log-Sobolev inequalities. In Section 4 we prove the main result and Corollaries 1 and 3. The rest of the paper deals with measures on the real line. One motivation is to make progress on a question that we do not fully settle: our main theorem shows an implication between two properties; are they actually equivalent? In Section 5, we recall the known criteria. In Section 6 we consider the weighted log-Sobolev inequalities used by [13] in order to derive Eq. 6. We show that the two properties are not equivalent. Workable criteria are available for measures on $\mathbb{R}$ with strictly increasing potential close to $\infty$. In the final section, we develop an elementary approach to deal with potential with vanishing derivatives or even decreasing parts. We illustrate this method on the functional inequalities of interest. 


\section{Preliminaries: a Few More Inequalities}

We start with the following observation.

Lemma 1 Suppose that a probability measure $\mu$ on $\mathbb{R}^{d}$ satisfies the Latata-Oleszkiewicz inequality (5) with constant $C_{L O}$. Then it satisfies the Poincaré inequality (2) with constant $C_{P}=C_{L O}$.

Proof By taking $\theta \rightarrow 1^{+}$in Eq. 5 we see that Eq. 2 holds for all positive smooth functions (with constant $C_{L O}$ ). Since the variance is translation invariant, we conclude that Eq. 2 holds for all smooth functions bounded from below. The general case follows by approximation.

Remark 1 Alternatively, one can deduce the Poincaré inequality from the fact that Inequality (5) implies dimension-free concentration and the results of [17].

For $r \in(1,2)$ denote

$$
F_{r}(t)=\log ^{2 / r^{\prime}}(1+t)-\log ^{2 / r^{\prime}}(2), \quad t \geq 0 .
$$

We say that a probability measure $\mu$ on $\mathbb{R}^{d}$ satisfies an $F_{r}$-Sobolev inequality if there exists $C$ such that for every smooth $g: \mathbb{R}^{d} \rightarrow \mathbb{R}$,

$$
\int_{\mathbb{R}^{d}} g^{2} F_{r}\left(\frac{g^{2}}{\int_{\mathbb{R}^{d}} g^{2} d \mu}\right) d \mu \leq C \int_{\mathbb{R}^{d}}|\nabla g|^{2} d \mu .
$$

This inequality is tight, i.e., we have equality for constant functions (if $g$ is constant and equal to zero on its support, then the expression $0 / 0$ should be interpreted as 0 here and in Eq. 11 below). We say that $\mu$ on $\mathbb{R}^{d}$ satisfies a defective $F_{r}$-Sobolev inequality if there exist $B$ and $C$ such that for every smooth $g: \mathbb{R}^{d} \rightarrow \mathbb{R}$,

$$
\int_{\mathbb{R}^{d}} g^{2} F_{r}\left(\frac{g^{2}}{\int_{\mathbb{R}^{d}} g^{2} d \mu}\right) d \mu \leq B \int_{\mathbb{R}^{d}} g^{2} d \mu+C \int_{\mathbb{R}^{d}}|\nabla g|^{2} d \mu .
$$

In [6] Barthe, Cattiaux, and Roberto provided capacity criteria for, among others, the Latała-Oleszkiewicz and $F_{r}$-Sobolev inequalities. We refer to Section 5 of [6] for a thorough overview of the topic, and in particular to the diagram on page 1041, which we use as a road map. The following theorem is a direct corollary of the results contained therein (and also in Wang's independent paper [26]).

Theorem 3 Let $r \in(1,2)$, and let $\mu$ be an absolutely continuous probability measure $\mu$ on $\mathbb{R}^{d}$. Assume that $\mu$ satisfies the Latata-Oleszkiewicz inequality (5) with parameter $r \in(1,2)$ and constant $C_{L O}$. Then $\mu$ satisfies the (tight) $F_{r}$-Sobolev inequality (9) with a constant $C \leq 1152 C_{L O}$.

Proof Denote $T(s):=s^{2(1-1 / r)}$. Recall the following definition of capacity: for Borel sets $A \subset \Omega \subset \mathbb{R}^{d}$, define

$$
\operatorname{Cap}_{\mu}(A, \Omega):=\inf \left\{\int_{\mathbb{R}^{d}}|\nabla f|^{2} d \mu: f_{\mid A} \geq 1 \text { and } f_{\mid \Omega^{c}}=0\right\}
$$


(the infimum is taken over all locally Lipschitz functions), and

$$
\operatorname{Cap}_{\mu}(A):=\inf \left\{\operatorname{Cap}_{\mu}(A, \Omega): A \subset \Omega \text { and } \mu(\Omega) \leq 1 / 2\right\} .
$$

Theorem 18 and Lemma 19 of [6] imply that if $\mu$ satisfies the Latała-Oleszkiewicz inequality (5) with some constant $C_{L O}$, then

$$
\mu(A) \frac{1}{T\left(\frac{1}{\log (1+1 / \mu(A))}\right)}=\mu(A) \log ^{2 / r^{\prime}}(1+1 / \mu(A)) \leq 6 C_{L O} \operatorname{Cap}_{\mu}(A)
$$

for every $A \subset \mathbb{R}^{d}$ with $\mu(A)<1 / 2$. Using $2 \log (1+t) \geq \log (1+2 t), t \geq 0$ and $r \in(1,2)$, we obtain that for all $A$ as above,

$$
\mu(A) \log ^{2 / r^{\prime}}(1+2 / \mu(A)) \leq 12 C_{L O} \operatorname{Cap}_{\mu}(A) .
$$

Theorem 28 of [6] then gives ${ }^{1}$ that for every smooth function $f: \mathbb{R}^{d} \rightarrow \mathbb{R}$,

$$
\int f^{2} \log ^{2 / r^{\prime}}\left(1+f^{2}\right) d \mu-\left(\int f^{2} d \mu\right) \log ^{2 / r^{\prime}}\left(1+\int f^{2} d \mu\right) \leq 1152 C_{L O} \int|\nabla f|^{2} d \mu .
$$

Substituting $f^{2}=g^{2} / \int_{\mathbb{R}^{d}} g^{2} d \mu$ yields the claimed $F_{r}$-Sobolev inequality (9).

Remark 2 The latter theorem remains valid even if $\mu$ is not absolutely continuous. To see this we use an approximation argument. Let $\gamma_{\varepsilon}$ be the centered Gaussian measure on $\mathbb{R}^{d}$ with covariance matrix $\varepsilon$ Id. For small enough $\varepsilon>0, \gamma_{\varepsilon}$ satisfies the Latała-Oleszkiewicz inequality with the same constant as $\mu$ and hence, by tensorization, so does $\mu \otimes \gamma_{\varepsilon}$. Testing the inequality with the function $(x, y) \mapsto f(x+y)$, we conclude that $\mu * \gamma_{\varepsilon}$ also satisfies the Latała-Oleszkiewicz inequality (with a constant at most $2 C_{L O}(\mu)$ when $\varepsilon$ is small enough). Thus, by Theorem 3, $\mu * \gamma_{\varepsilon}$ satisfies the $F_{r}$-Sobolev inequality. We fix a bounded smooth Lipschitz function, take $\varepsilon \rightarrow 0$, and arrive at the conclusion that $\mu$ satisfies the $F_{r}$-Sobolev inequality for all bounded smooth Lipschitz functions (we have pointwise convergence and since the function is Lipschitz and bounded we can use the dominated convergence theorem). Now if $f$ is an arbitrary smooth function such that $\int_{\mathbb{R}^{d}}|\nabla f|^{2} d \mu<\infty$, then it suffices to consider functions $f_{n}=\Psi_{n}(f)$, where $\Psi_{n}: \mathbb{R} \rightarrow \mathbb{R}$ is, say, an odd and non-decreasing function defined by

$$
\Psi_{n}(t)= \begin{cases}\Psi_{n}(-t) & \text { for } t<0, \\ t & \text { for } t \in[0, n), \\ \Psi_{n}(t)=n+\psi(t) & \text { for } t \in[n, n+2], \\ \Psi_{n}(t)=n+1 & \text { for } t>n+2,\end{cases}
$$

and $\psi:[0,2] \rightarrow[0,1]$ is smooth and increasing on $(0,2)$, such that $\psi(0)=0, \psi(2)=1$, $\psi^{\prime}(0+)=1, \psi^{\prime}(2-)=0, \psi(t) \leq t$ for $t \in[0,2]$. We then use dominated convergence on the right-hand side and monotone convergence on the left-hand side (note that by the Poincaré inequality $f$ is square-integrable).

\footnotetext{
${ }^{1}$ The assumption of absolute continuity of $\mu$ comes into play at this point. Indeed, the proof of Theorem 28 in [6] relies on a decomposition of $\mathbb{R}^{d}$ into level sets $\left\{f^{2}>\rho_{k}\right\}$, for some well chosen $\rho_{k}$ (cf. proof of Theorem 20 in [6]), and one needs to know that the sets $\left\{f^{2}=\rho_{k}\right\} \cap\{|\nabla f| \neq 0\}$ are negligible.
} 
We need another inequality introduced by Barthe and Kolesnikov in [3]. For $\tau \in(0,1)$, one says that a probability measure $\mu$ on $\mathbb{R}^{d}$ satisfies the inequality $I(\tau)$ if there exist constants $B_{1}$ and $C_{1}$ such that for every smooth $f: \mathbb{R}^{d} \rightarrow \mathbb{R}$,

$$
\operatorname{Ent}_{\mu}\left(f^{2}\right) \leq B_{1} \int_{\mathbb{R}^{d}} f^{2} d \mu+C_{1} \int_{\mathbb{R}^{d}}|\nabla f|^{2} \log ^{1-\tau}\left(e+\frac{f^{2}}{\int_{\mathbb{R}^{d}} f^{2} d \mu}\right) d \mu .
$$

This inequality is related to the previous ones. The next statement is a quotation of Theorem 4.1 in [3], with a stronger assumption (of a Poincaré inequality instead of a local Poincaré inequality)

Theorem 4 (Barthe-Kolesnikov [3]) Let $r \in(1,2)$. Let $\mu$ be a probability measure satisfying Inequality $I\left(2 / r^{\prime}\right)$. Then $\mu$ satisfies a defective $F_{r}$-Sobolev inequality (10) and a defective modified log-Sobolev inequality with parameter $r$.

If in addition $\mu$ satisfies a Poincaré inequality, then its satisfies an $F_{r}$-Sobolev inequality (9) and a modified log-Sobolev inequality with parameter $r$, Eq. 6, with constants depending only on the constants of the input inequalities.

We establish a partial converse to the above implication:

Theorem 5 Let $r \in(1,2)$. Assume that a probability measure $\mu$ on $\mathbb{R}^{d}$ satisfies the defective $F_{r}$-Sobolev inequality (10) with constants $B$ and $C$. Then $\mu$ satisfies the $I\left(2 / r^{\prime}\right)$ inequality (11) with some constants $B_{1}$ and $C_{1}$ which depend only on $B, C$, and $r$.

Proof We reverse the reasoning from the proof of Theorem 4.1 in [3]. Fix a smooth function $f$ such that the right-hand side of Eq. 11 is finite. We may and do assume that $\int_{\mathbb{R}^{d}} f^{2}\left|\ln \left(f^{2}\right)\right| d \mu<\infty .{ }^{2}$ Consider the function

$$
\Phi(x)=x^{2} \log ^{1-2 / r^{\prime}}\left(e+x^{2}\right), \quad x \in \mathbb{R},
$$

(which is convex since the function $t \mapsto t \log ^{1-2 / r^{\prime}}(e+t)$ is convex and increasing for $t>0$. Recall that $r^{\prime}>2$ ). Denote by $L$ the Luxemburg norm of $f$ related to $\Phi$ :

$$
L=\inf \left\{\lambda>0: \int_{\mathbb{R}^{d}} \Phi(f / \lambda) d \mu \leq 1\right\} .
$$

Note that $L<\infty, \int_{\mathbb{R}^{d}} \Phi(f / L) d \mu=1$ (by the definition of $L$ ), and $L^{2} \geq \int_{\mathbb{R}^{d}} f^{2} d \mu$ (since $\left.\Phi(x) \geq x^{2}\right)$.

Set $g:=\sqrt{\Phi(f / L)}$. We have $\int_{\mathbb{R}^{d}} g^{2} d \mu=1$ and Eq. 10 reads

$$
\int_{\mathbb{R}^{d}} g^{2}\left(\log ^{2 / r^{\prime}}\left(1+g^{2}\right)-\log ^{2 / r^{\prime}}(2)\right) d \mu \leq B \int_{\mathbb{R}^{d}} g^{2} d \mu+C \int_{\mathbb{R}^{d}}|\nabla g|^{2} d \mu .
$$

\footnotetext{
${ }^{2}$ Indeed, like above let us define odd and non-decreasing functions $\Psi_{n}: \mathbb{R} \rightarrow \mathbb{R}$ by putting $\Psi_{n}(t)=t$ for $t \in$ $[0, n), \Psi_{n}(t)=n+1$ for $t>n+2$; for $t \in[n, n+2]$ let us take $\Psi_{n}(t)=n+\psi(t)$, where $\psi:[0,2] \rightarrow[0,1]$ is smooth and increasing on $(0,2)$, and satisfies $\psi(0)=0, \psi(2)=1, \psi^{\prime}(0+)=1, \psi^{\prime}(2-)=0, \psi(t) \leq t$. Then the functions $f_{n}=\Psi_{n}(f)$ are smooth, bounded (and hence $\int_{\mathbb{R}^{d}} f_{n}^{2}\left|\ln \left(f_{n}^{2}\right)\right| d \mu<\infty$ ) and converge to $f$ pointwise. After proving that Eq. 11 holds for $f_{n}$, we obtain the assertion for $f$ by taking $n \rightarrow \infty$ and using monotone convergence on the left-hand side and the Lebesgue dominated convergence theorem on the right-hand side (note that we know that $f$ and $f_{n}$ are square-integrable, $\left|\nabla f_{n}\right|$ is up to a constant smaller than $|\nabla f|, f_{n}=f$ if $|f| \in[0, n],\left|f_{n}\right| \leq|f|$ if $|f| \in[n, n+2]$, and if $|f|>n+2$, then $\left.\nabla f_{n}=0\right)$.
} 
Let us first express the right-hand side of this inequality in terms of $f$. For $x \in \mathbb{R}$ denote $\varphi(x):=x \log ^{1 / 2-1 / r^{\prime}}\left(e+x^{2}\right)$. Then

$$
\begin{aligned}
0 \leq \varphi^{\prime}(x) & =\log ^{1 / 2-1 / r^{\prime}}\left(e+x^{2}\right)+\left(1 / 2-1 / r^{\prime}\right) \frac{2 x^{2}}{e+x^{2}} \log ^{-1 / 2-1 / r^{\prime}}\left(e+x^{2}\right) \\
& \leq 2 \log ^{1 / 2-1 / r^{\prime}}\left(e+x^{2}\right)
\end{aligned}
$$

and thus

$$
\begin{aligned}
|\nabla g|^{2}=\frac{|\nabla f|^{2}}{L^{2}}\left(\varphi^{\prime}(f / L)\right)^{2} & \leq 4 \frac{|\nabla f|^{2}}{L^{2}} \log ^{1-2 / r^{\prime}}\left(e+f^{2} / L^{2}\right) \\
& \leq 4 \frac{|\nabla f|^{2}}{L^{2}} \log ^{1-2 / r^{\prime}}\left(e+\frac{f^{2}}{\int_{\mathbb{R}^{d}} f^{2} d \mu}\right)
\end{aligned}
$$

Hence

$$
B \int_{\mathbb{R}^{d}} g^{2} d \mu+C \int_{\mathbb{R}^{d}}|\nabla g|^{2} d \mu \leq B+4 C \int_{\mathbb{R}^{d}} \frac{|\nabla f|^{2}}{L^{2}} \log ^{1-2 / r^{\prime}}\left(e+\frac{f^{2}}{\int_{\mathbb{R}^{d}} f^{2} d \mu}\right) d \mu .
$$

As for the left-hand side of Eq. 12, it is easy to see that there exists $\kappa_{1}=\kappa_{1}(r)>0$ such that, for $y>0$,

$$
y \log ^{1-2 / r^{\prime}}(e+y)\left(\log ^{2 / r^{\prime}}\left(1+y \log ^{1-2 / r^{\prime}}(e+y)\right)-\log ^{2 / r^{\prime}}(2)\right) \geq y \log (y)-\kappa_{1} .
$$

Applying this inequality with $y=f^{2} / L^{2}$, we arrive at

$$
\int_{\mathbb{R}^{d}} g^{2}\left(\log ^{2 / r^{\prime}}\left(1+g^{2}\right)-\log ^{2 / r^{\prime}}(2)\right) d \mu \geq \int_{\mathbb{R}^{d}} \frac{f^{2}}{L^{2}} \log \left(f^{2} / L^{2}\right) d \mu-\kappa_{1} .
$$

Together with Eq. 13 this yields

$$
\int_{\mathbb{R}^{d}} f^{2} \log \left(f^{2} / L^{2}\right) d \mu \leq\left(B+\kappa_{1}\right) L^{2}+4 C \int_{\mathbb{R}^{d}}|\nabla f|^{2} \log ^{1-2 / r^{\prime}}\left(e+\frac{f^{2}}{\int_{\mathbb{R}^{d}} f^{2} d \mu}\right) d \mu .
$$

It remains to replace the expression on the left-hand side by $\operatorname{Ent}_{\mu}\left(f^{2}\right)$ and estimate $L^{2}$.

Since

$$
\begin{aligned}
\operatorname{Ent}_{\mu}\left(f^{2}\right) & =\inf _{t>0} \int_{\mathbb{R}^{d}}\left(f^{2} \log \left(f^{2} / t\right)-f^{2}+t\right) d \mu \\
& \leq \int_{\mathbb{R}^{d}}\left(f^{2} \log \left(f^{2} / L^{2}\right)-f^{2}+L^{2}\right) d \mu
\end{aligned}
$$

we conclude that

$$
\operatorname{Ent}_{\mu}\left(f^{2}\right) \leq\left(B+\kappa_{1}+1\right) L^{2}+4 C \int_{\mathbb{R}^{d}}|\nabla f|^{2} \log ^{1-2 / r^{\prime}}\left(e+\frac{f^{2}}{\int_{\mathbb{R}^{d}} f^{2} d \mu}\right) d \mu .
$$

Finally, it is easy to see that for every $\varepsilon>0$ there exist $\kappa_{2}=\kappa_{2}(\varepsilon, r)$ such that, for $y>0$,

$$
y \log 1-2 / r^{\prime}(e+y) \leq \varepsilon y \log (y)+\kappa_{2}
$$

Using first the definition of $L$ and the fact that $L^{2} \geq \int_{\mathbb{R}^{d}} f^{2} d \mu$, and then the above bound (with $y=f^{2} / \int_{\mathbb{R}^{d}} f^{2} d \mu$ ) we can thus estimate

$$
\begin{aligned}
L^{2} & =\int_{\mathbb{R}^{d}} f^{2} \log ^{1-2 / r^{\prime}}\left(e+f^{2} / L^{2}\right) d \mu \leq \int_{\mathbb{R}^{d}} f^{2} \log ^{1-2 / r^{\prime}}\left(e+\frac{f^{2}}{\int_{\mathbb{R}^{d}} f^{2} d \mu}\right) d \mu \\
& \leq \varepsilon \operatorname{Ent}_{\mu}\left(f^{2}\right)+\kappa_{2} \int_{\mathbb{R}^{d}} f^{2} d \mu .
\end{aligned}
$$


Eventually, for $\varepsilon$ small enough we combine this bound with Eq. 14 and simplify the entropy terms (recall that by our assumption $\operatorname{Ent}_{\mu}\left(f^{2}\right)<\infty$ ) in order to reach the claim.

\section{Proof of the Main Result and Its Corollaries}

Proof of Theorem 2 Our assumption is that $\mu$ satisfies a Latała-Oleszkiwicz inequality with parameter $r$. Therefore by Lemma 1 it also satisfies a Poincaré inequality, and by Theorem 3 (and Remark 2 if $\mu$ is not absolutely continuous) it satisfies a (tight) $F_{r}$-Sobolev inequality. From Theorem 5, we deduce that $\mu$ enjoys an $I\left(2 / r^{\prime}\right)$-inequality. Eventually Theorem 4 asserts that the $I\left(2 / r^{\prime}\right)$, together with the Poincaré inequality, implies a (tight) modified logSobolev inequality with parameter $r$. The constants that we obtain in the above inequalities only depend on $r$ and $C_{L O(r)}$. Proving the claimed dependence in $C_{L O(r)}$ is straightforward, but requires to track the constants in the various intermediate statements. We omit the details.

Remark 3 Let us comment here that for $d=1$ it is known that the inequalities Eqs. 5 and 6 hold if and only if they hold with the integration with respect to $\mu$ on the right-hand side replaced by integration with respect to $\mu_{a c}$, the absolutely continuous part of $\mu$ (cf. [8], Appendix of [20], Appendix of [16]).

For the proofs of the corollaries we need one more technical lemma. We denote by $H_{r^{\prime}}^{*}(t):=\sup _{s \in \mathbb{R}}\left\{s t-H_{r^{\prime}}(s)\right\}, t \in \mathbb{R}$, the Legendre transform of $H_{r^{\prime}}$ (we refer to the book [21] for more information on this topic).

Lemma 2 Let $r \in(1,2)$. The function $H_{r^{\prime}}^{*}$ is given by the formula

$$
H_{r^{\prime}}^{*}(t)= \begin{cases}t^{2} / 4 & \text { if } 0 \leq|t| \leq 2 \\ |t|-1 & \text { if } 2 \leq|t| \leq r^{\prime} \\ \frac{1}{r-1}\left(\frac{1}{r^{\prime}}|t|\right)^{r} & \text { if }|t| \geq r^{\prime}\end{cases}
$$

Moreover, $H_{r^{\prime}}^{*}(t) \geq \frac{1}{4} \min \left\{t^{2},|t|^{r}\right\}$.

Proof The first part is a straightforward calculation. To prove the second part, first notice that

$$
\inf _{r \in(1,2)} \frac{1}{r-1}\left(\frac{1}{r^{\prime}}\right)^{r}=1 / 4 .
$$

This allows to verify the inequality $H_{r^{\prime}}^{*}(t) \geq \frac{1}{4} \min \left\{t^{2},|t|^{r}\right\}$ for $t \in[0,2] \cup\left[r^{\prime}, \infty\right)$. It remains to prove the inequality on the interval $\left[2, r^{\prime}\right]$, where $H_{r^{\prime}}^{*}(t)=t-1$ is affine in $t$, and $\frac{1}{4} \min \left\{t^{2},|t|^{r}\right\}=\frac{1}{4} t^{r}$ is convex in $t$. Therefore it is enough to check the inequality at the endpoints of the interval, which we have already done.

Proof of Corollary 1 A classical argument of Herbst (see, e.g., [19]) allows to deduce concentration bounds from log-Sobolev inequalities. It was implemented in [5] for modified $\log$-Sobolev inequalities with energy terms $H(\nabla f / f)$ involving general functions $H$. We 
rather follow the calculation of [1] which is more suited to the case $H=H_{r^{\prime}}$. Take a function $f: \mathbb{R}^{d n} \rightarrow \mathbb{R}$ and denote

$$
A=\sup _{x \in \mathbb{R}^{d n}}|\nabla f(x)|, \quad B=\sup _{x \in \mathbb{R}^{d \cdot n}}\|\nabla f(x)\|_{r^{\prime}, 2} .
$$

Moreover, let $F(\lambda)=\int_{\mathbb{R}^{d n}} e^{\lambda f(x)} d \mu^{\otimes n}$. Then

$$
\lambda F^{\prime}(\lambda)=\int_{\mathbb{R}^{d n}} \lambda f(x) e^{\lambda f(x)} d \mu^{\otimes n}
$$

and hence, since $\mu$ satisfies the modified $\log$-Sobolev inequality with some constant $C=$ $C\left(C_{L O}, r\right)$ (by Theorem 2$)$ and by the tensorization property,

$$
\begin{aligned}
\lambda F^{\prime}(\lambda)-F(\lambda) \log F(\lambda) & =\operatorname{Ent}_{\mu^{\otimes n}}\left(e^{\lambda f}\right) \\
& \leq C \int_{\mathbb{R}^{d n}} \sum_{i=1}^{n} H_{r^{\prime}}\left(\frac{\lambda}{2}\left|\nabla_{i} f\right|\right) e^{\lambda f} d \mu^{\otimes n} \\
& \leq 2 C \max \left\{(A \lambda / 2)^{2},(B \lambda / 2)^{r^{\prime}}\right\} F(\lambda),
\end{aligned}
$$

where we used the inequality $\sum_{i} \max \left\{a_{i}^{2}, b_{i}^{r^{\prime}}\right\} \leq 2 \max \left\{\sum_{i} a_{i}^{2}, \sum_{i} b_{i}^{r^{\prime}}\right\}$. After dividing both sides by $\lambda^{2} F(\lambda)$ we can rewrite this as

$$
\left(\frac{1}{\lambda} \log F(\lambda)\right)^{\prime} \leq 2 C \max \left\{(A \lambda / 2)^{2},(B \lambda / 2)^{r^{\prime}}\right\} / \lambda^{2} .
$$

Since the right-hand side is an increasing function of $\lambda>0$ and

$$
\lim _{\lambda \rightarrow 0^{+}} \frac{1}{\lambda} \log F(\lambda)=\int_{\mathbb{R}^{d n}} f d \mu^{\otimes n},
$$

we deduce from the last inequality that

$$
\frac{1}{\lambda} \log F(\lambda) \leq \int_{\mathbb{R}^{d n}} f d \mu^{\otimes n}+2 C \max \left\{(A \lambda / 2)^{2},(B \lambda / 2)^{r^{\prime}}\right\} / \lambda,
$$

which is equivalent to

$$
\int_{\mathbb{R}^{d n}} e^{\lambda f} d \mu^{\otimes n} \leq \exp \left(\lambda \int_{\mathbb{R}^{d n}} f d \mu^{\otimes n}+2 C \max \left\{(A \lambda / 2)^{2},(B \lambda / 2)^{r^{\prime}}\right\}\right) .
$$

Therefore from Chebyshev's inequality we get, for $t>0$ and any $\lambda>0$,

$$
\begin{aligned}
\mu^{\otimes n}\left(f \geq \int_{\mathbb{R}^{d n}} f d \mu^{\otimes n}+t\right) & \leq \frac{\int_{\mathbb{R}^{d n}} e^{2 \lambda f} d \mu^{\otimes n}}{\exp \left(2 \lambda \int_{\mathbb{R}^{d n}} f d \mu^{\otimes n}+2 \lambda t\right)} \\
& \leq \exp \left(-2 \lambda t+2 C \max \left\{(A \lambda)^{2},(B \lambda)^{r^{\prime}}\right\}\right) .
\end{aligned}
$$

Now we can optimize the right-hand side with respect to $\lambda$. Let $U$ and $V$ be such that $A=U^{1 / 2} V, B=U^{1 / r^{\prime}} V$. We have

$$
\left.\max \left\{(A \lambda)^{2},(B \lambda)^{r^{\prime}}\right\}\right)=U \max \left\{(V \lambda)^{2},(V \lambda)^{r^{\prime}}\right\}=U H_{r^{\prime}}(V \lambda)
$$


and hence

$$
\mu^{\otimes n}\left(f \geq \int_{\mathbb{R}^{d n}} f d \mu^{\otimes n}+t\right) \leq \exp \left(-2 C U H_{r^{\prime}}^{*}\left(\frac{t}{C U V}\right)\right) .
$$

Using Lemma 2 and the definitions of $U$ and $V$ we get

$$
\mu^{\otimes n}\left(f \geq \int_{\mathbb{R}^{d n}} f d \mu^{\otimes n}+t\right) \leq \exp \left(-\frac{1}{2} \min \left\{\frac{t^{2}}{C A^{2}}, \frac{t^{r}}{C^{r-1} B^{r}}\right\}\right),
$$

which yields the assertion of the corollary.

Proof of Corollary 2 Let $f_{\varepsilon}$ be the convolution of $f$ and a Gaussian kernel, i.e., $f_{\varepsilon}(x)=$ $\mathbb{E} f(x+\sqrt{\varepsilon} G)$, where $G \sim \mathcal{N}(0, I)$. This function clearly inherits from $f$ the estimates of the Lipschitz constants. Since it is smooth, the $\ell_{2}$-norm and the norm $\|\cdot\|_{r^{\prime}, 2}$ of its gradient can be estimated pointwise by $L_{2}$ and $L_{r, 2}$, respectively. Therefore we can apply Corollary 1 to $f_{\varepsilon}$. Moreover, $\left|f_{\varepsilon}(x)-f(x)\right| \leq L_{2} \sqrt{\varepsilon} \mathbb{E}|G|$ and hence $f_{\varepsilon}$ converges uniformly to $f$ as $\varepsilon$ tends to zero. This observation ends the proof of the corollary.

Proof of Corollary 3 We follow the approach of Bobkov and Ledoux from Section 2 of [9]. Take a set $A \subset \mathbb{R}^{d n}$ with $\mu^{\otimes n}(A) \geq 1 / 2$. For $x=\left(x_{1}, \ldots, x_{n}\right) \in \mathbb{R}^{d \cdot n}$, denote

$$
F_{A}(x)=\inf _{a \in A} \sum_{i=1}^{n} \min \left\{\left|x_{i}-a_{i}\right|^{2},\left|x_{i}-a_{i}\right|^{r}\right\}
$$

(note that here $|\cdot|$ is the $\ell_{2}$-norm on $\mathbb{R}^{d}$ ). Take any $t>0$ and set $f=\min \left\{F_{A}, t\right\}$. We claim that for all $x, y \in \mathbb{R}^{d \cdot n}$,

$$
|f(x)-f(y)| \leq 2 \sqrt{t}|x-y|, \quad|f(x)-f(y)| \leq 2 t^{1 / r^{\prime}}\|x-y\|_{r, 2} .
$$

Suppose that we already know that this holds. Note that $\left(2 t^{1 / r^{\prime}}\right)^{r}=2^{r} t^{r-1}$. Also, $\int_{\mathbb{R}^{d n}} f d \mu^{\otimes n} \leq t / 2$ since $F_{A}=0$ on $A$ and $\mu^{\otimes n}(A) \geq 1 / 2$. Consequently, by Corollary 2 and Eq. 15,

$$
\begin{aligned}
\mu^{\otimes n}\left(F_{A} \geq t\right) & \leq \mu^{\otimes n}(f \geq t) \leq \mu^{\otimes n}\left(f \geq \int_{\mathbb{R}^{d n}} f d \mu^{\otimes n}+t / 2\right) \\
& \leq \exp \left(-\frac{1}{2} \min \left\{\frac{(t / 2)^{2}}{4 t C}, \frac{(t / 2)^{r}}{2^{r} t^{r-1} C^{r-1}}\right\}\right) \\
& =\exp \left(-\frac{t}{2} \min \left\{\frac{1}{16 C}, \frac{1}{4^{r} C^{r-1}}\right\}\right) \leq \exp (-K t),
\end{aligned}
$$

where $K=\frac{1}{32} \min \left\{1 / C, 1 / C^{r-1}\right\}$ and $C$ is the constant with which, by Theorem 2 , the modified log-Sobolev inequality holds for $\mu$. Since clearly

$$
\left\{F_{A}<t\right\} \subset A+\left\{\left(x_{1}, \ldots, x_{n}\right) \in \mathbb{R}^{d \cdot n}: \sum_{i=1}^{n} \min \left\{\left|x_{i}\right|^{2},\left|x_{i}\right|^{r}\right\} \leq t\right\},
$$

this yields the first assertion of the corollary. The second part follows by the inclusion

$$
\left\{\left(x_{1}, \ldots, x_{n}\right) \in \mathbb{R}^{d \cdot n}: \sum_{i=1}^{n} \min \left\{\left|x_{i}\right|^{2},\left|x_{i}\right|^{r}\right\} \leq t\right\} \subset \sqrt{t} B_{2}^{d n}+t^{1 / r} B_{r, 2}^{n, d} .
$$


It remains to prove the claim Eq. 15. To this end, consider the functions

$$
G(x)=\sum_{i=1}^{n} \min \left\{\left|x_{i}\right|^{2},\left|x_{i}\right|^{r}\right\}
$$

and $g(x)=\min \{G, t\}$. Since $g$ is locally Lipschitz it suffices to show that, a.e.,

$$
\sum_{i=1}^{n}\left|\nabla_{i} g\right|^{2} \leq 4 t, \quad \sum_{i=1}^{n}\left|\nabla_{i} g\right|^{r^{\prime}} \leq 2^{r^{\prime}} t .
$$

Indeed, this will imply that Eq. 15 holds with $g$ in place of $f$ (note that the norm $\|\cdot\|_{r, 2}$ is dual to the norm $\left.\|\cdot\|_{r^{\prime}, 2}\right)$. Since $f(x)=\inf _{a \in A} g(x-a)$ (and the infimum of Lipschitz functions is Lipschitz with the same constant), the same estimates will be inherited by $f$.

On the open set $\{G>t\}$ the estimates obviously hold, since $g$ is constant. The set $\{G=t\}$ is Lebesgue negligible. Thus in what follows it suffices to consider the set $\{G<t\}$ on which $g=G$.

If, for some $i,\left|x_{i}\right|<1$, then

$$
\begin{aligned}
& \left|\nabla_{i} g(x)\right|^{2}=4\left|x_{i}\right|^{2}=4 \min \left\{\left|x_{i}\right|^{2},\left|x_{i}\right|^{r}\right\}, \\
& \left|\nabla_{i} g(x)\right|^{r^{\prime}}=2^{r^{\prime}}\left|x_{i}\right|^{r^{\prime}} \leq 2^{r^{\prime}}\left|x_{i}\right|^{2}=2^{r^{\prime}} \min \left\{\left|x_{i}\right|^{2},\left|x_{i}\right|^{r}\right\} .
\end{aligned}
$$

If on the other hand $\left|x_{i}\right|>1$, then

$$
\begin{aligned}
& \left|\nabla_{i} g(x)\right|^{2}=r^{2}\left|x_{i}\right|^{2(r-1)} \leq 4\left|x_{i}\right|^{r}=4 \min \left\{\left|x_{i}\right|^{2},\left|x_{i}\right|^{r}\right\}, \\
& \left|\nabla_{i} g(x)\right|^{r^{\prime}}=r^{r^{\prime}}\left|x_{i}\right|^{r}=r^{r^{\prime}} \min \left\{\left|x_{i}\right|^{2},\left|x_{i}\right|^{r}\right\} .
\end{aligned}
$$

Thus, a.e. (the set where $\left|x_{i}\right|=1$ for some $i$ is negligible),

$$
\begin{aligned}
\left|\nabla_{i} g(x)\right|^{2} & \leq 4 \min \left\{\left|x_{i}\right|^{2},\left|x_{i}\right|^{r}\right\}, \\
\left|\nabla_{i} g(x)\right|^{r^{\prime}} & \leq 2^{r^{\prime}} \min \left\{\left|x_{i}\right|^{2},\left|x_{i}\right|^{r}\right\} .
\end{aligned}
$$

Consequently, on the set $\{G<t\}$, it holds a.e.

$$
\begin{aligned}
& \sum_{i=1}^{n}\left|\nabla_{i} g(x)\right|^{2} \leq 4 G(x) \leq 4 t, \\
& \sum_{i=1}^{n}\left|\nabla_{i} g(x)\right|^{r^{\prime}} \leq 2^{r^{\prime}} G(x) \leq 2^{r^{\prime}} t .
\end{aligned}
$$

Therefore the proof is complete.

\section{Criteria for Measures on the Real Line}

From now on we restrict to probability measures on the real line. In this setting, more tools are available. For several functional inequalities, workable equivalent criteria are available. They are based on Hardy type inequalities, of the form

$$
\int_{\mathbb{R}^{+}}|f-f(0)|^{p} d \mu \leq A \int_{\mathbb{R}^{+}}\left|f^{\prime}\right|^{p} d \nu .
$$

We refer to [2] for the history of the topic, from the original book of Hardy, Littlewood and Pólya, to the general version by Muckenhoupt. The textbook [2] also mentions that such Hardy inequalities yield the following criterion for Poincaré inequalities on $\mathbb{R}$ (where we include a numerical improvement from [20]): 
Let $\mu$ be a probability measure on $\mathbb{R}$, with median $m$. Let $v$ be a probability measure on $\mathbb{R}$, and let $n$ denote the density of its absolutely continuous part. Then the (possibly infinite) best constant $C_{P}$ such that for all smooth $f$,

$$
\operatorname{Var}_{\mu}(f) \leq C_{P} \int_{\mathbb{R}}\left(f^{\prime}\right)^{2} d v
$$

verifies $\max \left(B_{P}^{+}, B_{P}^{-}\right) \leq C_{P} \leq 4 \max \left(B_{P}^{+}, B_{P}^{-}\right)$, with

$$
B_{P}^{+}=\sup _{x>m} \mu([x,+\infty)) \int_{m}^{x} \frac{1}{n}, \quad B_{P}^{-}=\sup _{x<m} \mu((-\infty, x]) \int_{x}^{m} \frac{1}{n},
$$

where by convention $0 \cdot \infty=0$.

Bobkov and Götze [8] extended the reach of these methods, by proving a similar statement for log-Sobolev inequalities of the form

$$
\operatorname{Ent}_{\mu}\left(f^{2}\right) \leq C_{L S} \int_{\mathbb{R}}\left(f^{\prime}\right)^{2} d \nu
$$

Their result reads as the previous one, with different numerical constants and $B_{P}^{+}, B_{P}^{-}$ replaced by

$$
B_{L S}^{+}=\sup _{x>m} \mu([x,+\infty)) \log \left(\frac{1}{\mu([x,+\infty))}\right) \int_{m}^{x} \frac{1}{n},
$$

and $B_{L S}^{-}$defined similarly for $x<m$. This criterion was later extended to the LatałaOleszkiewicz inequality (5). Let $\mu$ be a probability measure on $\mathbb{R}$. Denote by $m$ the median of $\mu$ and by $n$ the density of its absolutely continuous part. Barthe and Roberto [4] proved that $\mu$ satisfies the Latała-Oleszkiewicz inequality (5) if and only if $\max \left\{B_{L O(r)}^{+}, B_{L O(r)}^{-}\right\}<$ $\infty$, where

$$
B_{L O(r)}^{+}:=\sup _{x>m} \mu([x, \infty)) \log ^{2 / r^{\prime}}\left(1+\frac{1}{2 \mu([x, \infty))}\right) \int_{m}^{x} \frac{1}{n(t)} d t
$$

and $B_{L O(r)}^{-}$is defined similarly but with $x<m$. Moreover the best possible constant $C_{L O(r)}$ in Eq. 5 is comparable to $\max \left\{B_{L O(r)}^{+}, B_{L O(r)}^{-}\right\}$, up to numerical constants which do not depend on $r \in(1,2)$.

In the subsequent paper [5], Barthe and Roberto provided a criterion for the modified logSobolev inequality (6). However, they did not reach a full equivalence. Here is the outline of Theorem 10 in [5]. Let $d \mu(t)=n(t) d t$ be a probability measure on $\mathbb{R}$ with median $m$. If $\mu$ satisfies the Poincaré inequality with constant $C_{P}$ and $\max \left\{B_{m L S(r)}^{+}, B_{m L S(r)}^{-}\right\}<\infty$, where

$$
B_{m L S(r)}^{+}:=\sup _{x>m} \mu([x, \infty)) \log \left(\frac{1}{\mu([x, \infty))}\right)\left(\int_{m}^{x} \frac{1}{n(t)^{r-1}} d t\right)^{1 /(r-1)}
$$

and $B_{m L S}^{-}$is defined similarly but with $x<m$, then $\mu$ satisfies the modified log-Sobolev inequality (6) with constant

$$
C_{m L S} \leq 235 C_{P}+2^{r^{\prime}+1} \max \left\{B_{m L S(r)}^{+}, B_{m L S(r)}^{-}\right\} .
$$


The converse implication is, so far, known only under the following additional assumption: there exists $\varepsilon>0$ such that for all $x \neq m$

$$
\frac{1}{n(x)^{r-1}} \geq \varepsilon \int_{\min (m, x)}^{\max (m, x)} \frac{1}{n^{r-1}} .
$$

In this case, if $\mu$ satisfies the modified log-Sobolev inequality (6), then

$$
\max \left\{B_{m L S(r)}^{+}, B_{m L S(r)}^{-}\right\}<\infty
$$

and this quantity can be estimated in terms of the constant $C_{m L S(r)}$ up to constants depending on $r$ and $\varepsilon$. The Poincaré inequality is a classical consequence of the modified $\log$-Sobolev inequality, exactly as in Lemma 1.

Even though the above criteria involve simple concrete quantities, it does not seem easy to use them in order to reprove our main result Theorem 2 for measures on $\mathbb{R}$. However, if one assumes for example that $d \mu(x)=\exp (-V(x)) d x, x \in \mathbb{R}$, where $V$ is symmetric, of class $C^{2}, \liminf _{x \rightarrow \infty} V^{\prime}(x)>0$, and

$$
\lim _{x \rightarrow \infty} \frac{V^{\prime \prime}(x)}{V^{\prime}(x)^{2}}=0
$$

then one can estimate the quantities $B_{L O(r)}^{+}, B_{m L S(r)}^{+}$and show that the Latała-Oleszkiewicz inequality (5) is equivalent to the modified $\log$-Sobolev inequality (6) and furthermore to the condition

$$
\limsup _{x \rightarrow \infty} \frac{V(x)}{V^{\prime}(x)^{r^{\prime}}}<\infty
$$

(see Remark 21 in [5]).

In the rest of the paper we use and develop one-dimensional criteria in order to study whether the modified log-Sobolev inequality is actually equivalent to other inequalities which are known to imply it.

\section{Weighted vs. Modified Log-Sobolev Inequality}

It is known that if a probability measure $\mu$ on $\mathbb{R}^{d}$ satisfies a certain weighted log-Sobolev inequality (and an integrability condition), then it also satisfies a modified log-Sobolev inequality, see Theorem 3.4 in [12] (in the context of a specific measure on the real line a similar argument appears already in the large entropy case of the proof of Theorem 3.1 from [13]). The goal of this subsection is to show that the converse implication does not hold in general, even for measures on the real line.

First we present a workable criterion for the weighted log-Sobolev inequality.

Proposition 1 Let $d \mu(x)=e^{-V(x)} d x$ be a probability measure on the real line. Let $V: \mathbb{R} \rightarrow \mathbb{R}$ be even and locally bounded. Assume that in some neighborhood of $\infty$, the function $V$ is of class $C^{2}$, and that

(i) $\liminf _{x \rightarrow \infty} V^{\prime}(x)>0$,

(ii) $\lim _{x \rightarrow \infty} \frac{V^{\prime \prime}(x)}{V^{\prime}(x)^{2}}=0$. 
Then, there exists $C<\infty$ such that $\mu$ satisfies the following weighted log-Sobolev inequality: for every $f: \mathbb{R} \rightarrow \mathbb{R}$,

$$
\operatorname{Ent}_{\mu}\left(f^{2}\right) \leq C \int_{\mathbb{R}} f^{\prime}(x)^{2}\left(1+|x|^{2-r}\right) d \mu(x),
$$

if and only if

$$
\limsup _{x \rightarrow \infty} \frac{V(x)}{|x|^{2-r} V^{\prime}(x)^{2}}<\infty
$$

Remark 4 Condition $(i i)$ can be weakened to $\lim \sup _{x \rightarrow \infty} \frac{\left|V^{\prime \prime}(x)\right|}{V^{\prime}(x)^{2}}<1$.

Proof of Proposition 1 Denote $W(x):=V(x)-\log \left(1+|x|^{2-r}\right), x \in \mathbb{R}$. By the BobkovGötze criterion [8] (see Eq. 17), $\mu$ satisfies the weighted log-Sobolev inequality if and only if

$$
\sup _{x>0} \mu((x, \infty)) \log \left(\frac{1}{\mu((x, \infty))}\right) \int_{0}^{x} e^{W(t)} d t<\infty .
$$

Of course, it suffices to investigate what happens for $x \rightarrow \infty$. Note that

$$
\liminf _{x \rightarrow \infty} W^{\prime}(x)=\liminf _{x \rightarrow \infty}\left(V^{\prime}(x)-\frac{(2-r) x^{1-r}}{1+x^{2-r}}\right)>0
$$

(by Assumption (i)) and

$$
\lim _{x \rightarrow \infty} \frac{W^{\prime \prime}(x)}{W^{\prime}(x)^{2}}=0
$$

(by (ii) and the fact that $\left.W^{\prime \prime}(x)=V^{\prime \prime}(x)+o(1)\right)$. Thus, as $x \rightarrow \infty$,

$$
\begin{aligned}
\mu((x, \infty))=\int_{x}^{\infty} e^{-V(t)} d t & \sim \frac{e^{-V(x)}}{V^{\prime}(x)}, \\
\int_{0}^{x} e^{W(t)} d t & \sim \frac{e^{W(x)}}{W^{\prime}(x)}=\frac{e^{V(x)}}{\left(1+|x|^{2-r}\right)\left(V^{\prime}(x)+o(1)\right)}
\end{aligned}
$$

(here by ' $\sim$ ' we mean that the ratio of both sides tends to 1 as $x \rightarrow \infty$; to prove that this is indeed the case it suffices to consider the ratio of the derivatives of both sides). Therefore, Eq. 23 holds if and only if

$$
\limsup _{x \rightarrow \infty} \frac{V(x)+\log V^{\prime}(x)}{\left(1+|x|^{2-r}\right)\left(V^{\prime}(x)+o(1)\right) V^{\prime}(x)}<\infty,
$$

which, since $V^{\prime}(x)$ is bounded away from zero as $x \rightarrow \infty$, happens if and only if

$$
\limsup _{x \rightarrow \infty} \frac{V(x)}{|x|^{2-r} V^{\prime}(x)^{2}}<\infty
$$

This finishes the proof.

Our example is a modification of the example constructed by Cattiaux and Guillin [11] to prove that the log-Sobolev inequality is strictly stronger than Talagrand's transportation cost inequality. 
Proposition 2 For $r \in(1,2)$ and $\max \{r / 2, r-1 / r\}<\beta-1<r-1 / 2$ define

$$
U(x)=U_{r, \beta}(x)=|x|^{r+1}+(r+1)|x|^{r} \sin ^{2}(x)+|x|^{\beta}, \quad x \in \mathbb{R} .
$$

Let $\mu_{r, \beta}$ be the probability measure with density proportional to $e^{-U_{r, \beta}(x)}$. Then $\mu_{r, \beta}$ satisfies the modified log-Sobolev inequality (6) and the Latata-Oleszkiewicz inequality (5) (with $d=1$ ).

On the other hand, $\mu_{r, \beta}$ does not satisfy the weighted log-Sobolev inequality (22).

Proof Let us first note that $\beta \in(r, r+1)$. For $x>0$,

$$
\begin{aligned}
U(x) & =x^{r+1}+(r+1) x^{r} \sin ^{2}(x)+x^{\beta}, \\
U^{\prime}(x) & =(r+1)(1+\sin (2 x)) x^{r}+(r+1) r x^{r-1} \sin ^{2}(x)+\beta x^{\beta-1} .
\end{aligned}
$$

Clearly, $U^{\prime}(x) \geq \beta x^{\beta-1}$; in particular $\liminf _{x \rightarrow \infty} U^{\prime}(x)>0$. Moreover, for $x>1,\left|U^{\prime \prime}(x)\right|$ can be bounded by $M x^{r}$ for some constant $M=M(r, \beta)$. Thus,

$$
\lim _{x \rightarrow \infty} \frac{\left|U^{\prime \prime}(x)\right|}{U^{\prime}(x)^{2}} \leq \lim _{x \rightarrow \infty} \frac{M x^{r}}{\beta^{2} x^{2(\beta-1)}}=0,
$$

since $\beta-1>r / 2$. We are thus in position to apply workable versions of the criteria for the modified and weighted log-Sobolev inequalities (note that the normalization of $\mu_{r, \beta}$ amounts to adding a constant to the potential $U$, which does not affect the calculations and reasoning below).

First note that

$$
\lim _{x \rightarrow \infty} \frac{U(x)}{U^{\prime}(x)^{r^{\prime}}} \leq \lim _{x \rightarrow \infty} \frac{(r+3) x^{r+1}}{\beta^{r^{\prime}} x^{(\beta-1) r^{\prime}}}=0,
$$

since $(\beta-1) r^{\prime}>(r-1 / r) r^{\prime}=r+1$. Thus, by the Barthe-Roberto criterion (see Eq. 21), $\mu_{r, \beta}$ satisfies the modified log-Sobolev and the Latała-Oleszkiewicz inequality.

On the other hand, for certain values of $x \rightarrow \infty$ (e.g., for $x=k \pi-\pi / 4, k \in \mathbb{N}$ ), we have $\left|U^{\prime}(x)\right| \leq((r+1) r+\beta) x^{\beta-1}$. Hence

$$
\limsup _{x \rightarrow \infty} \frac{U(x)}{x^{2-r} U^{\prime}(x)^{2}} \geq \lim _{x \rightarrow \infty} \frac{x^{r+1}}{x^{2-r}((r+1) r+\beta)^{2} x^{2(\beta-1)}}=\infty,
$$

since $\beta-1<r-1 / 2$. Thus, by Proposition 1 above, $\mu_{r, \beta}$ cannot satisfy the weighted log-Sobolev inequality.

Remark 5 The introduction of [22], suggests that the results of our Theorem 2 are contained in [27], namely that it follows from [27] that the $F_{r}$-Sobolev inequality (9) implies the modified log-Sobolev inequality (6). We would like to rectify this: Wang's paper [27] deals with measures with faster decay than Gaussian. He proves that in that setting an appropriate super Poincaré inequality (or equivalently, an appropriate $F$-Sobolev inequality) implies a certain weighted log-Sobolev inequality. However, in our setting (measures with tail decay slower than Gaussian), we have an example of a measure which satisfies the modified log-Sobolev inequality (6) and the Latała-Oleszkiewicz inequality (5) (or equivalently, the $F_{r}$-Sobolev inequality (9)), but does not satisfy the weighted log-Sobolev inequality (22). Therefore Theorem 2 cannot be deduced from Wang's paper [27]. 


\section{On Potentials with Vanishing Derivatives}

\subsection{Motivation}

Recall that $r \in(1,2)$ is the parameter associated with the Latała-Oleszkiewicz inequality (5) and the modified log-Sobolev inequality (6). Throughout this section we consider symmetric probability measures on the real line of the form

$$
d \mu(x)=d \mu_{V}(x)=\frac{1}{Z} \exp (-V(x)) d x, \quad x \in \mathbb{R},
$$

where $V: \mathbb{R} \rightarrow \mathbb{R}$ is even and $Z$ is the normalization constant.

It is easy to see that if $\varepsilon \in[0,1)$ and for $x \in \mathbb{R}$

$$
V(x)=|x+\varepsilon \sin (x)|^{r}, \quad x>0,
$$

then $\mu_{V}$ satisfies both the Latała-Oleszkiewicz inequality (5) and the modified logSobolev inequality (6). Indeed, if $\varepsilon \in[0,1)$, then $\liminf _{x \rightarrow+\infty} V^{\prime}(x)>0$, $\lim _{x \rightarrow \infty} V^{\prime \prime}(x) / V^{\prime}(x)^{2}=0$ and the claim follows from the simplified versions of the Barthe-Roberto criteria (see Eq. 21).

This example becomes more interesting for $\varepsilon=1$ : since for any integer $k, V^{\prime}((2 k+$ $1) \pi)=0$ we cannot apply the simplified asymptotic versions of the criteria. In particular, one would like to know if, for measures with such potentials, the modified log-Sobolev inequality (6) and the Latała-Oleszkiewicz inequality (5) are valid simultaneously.

In the limit case $r=2$, Cattiaux [10] proved that if

$$
V(x)=x^{2}+2 \lambda x \sin (x), \quad x>0,
$$

then $\mu_{V}$ satisfies the classical log-Sobolev inequality if and only if $|\lambda|<1$ (note that this potential differs from $(x+\lambda \sin (x))^{2}$ only by a bounded perturbation). He used probabilistic methods which seem to rely on the fact that $r=2$. Below we present an analytic approach and obtain an extension of his results.

\subsection{Results}

For $\alpha>1$ define

$$
V_{\alpha}(x)=|x+\sin (x)|^{\alpha}, \quad x \in \mathbb{R} .
$$

Let $v_{\alpha}$ be the probability measure with density proportional to $\exp \left(-V_{\alpha}\right)$ :

$$
d v_{\alpha}(x)=\frac{1}{Z_{\alpha}} \exp \left(-V_{\alpha}(x)\right) d x, \quad x \in \mathbb{R} .
$$

Proposition 3 Let $\alpha>1$ and $r \in(1,2)$. The following assertions are equivalent

(i) $r \leq r_{0}(\alpha):=\frac{3 \alpha}{2 \alpha+1}$,

(ii) $v_{\alpha}$ satisfies the Latata-Oleszkiewicz inequality (5) with parameter $r$,

(iii) $v_{\alpha}$ satisfies the modified log-Sobolev inequality (6) with parameter $r$.

Remark 6 For Cattiaux's example the threshold is $r_{0}(2)=6 / 5$.

The threshold $r_{0}(\alpha)$ in Inequalities Eqs. 5 and 6 together with Corollary 3 might at first suggest that the measures $v_{\alpha}$ exhibit weaker concentration properties than one would 
expect given the decay of their densities (note that $r_{0}(\alpha)<\alpha$ ). In order to get two-level concentration with the expected rate one can use transportation cost inequalities, see [14, $24,25]$.

Let $\alpha \in(1,2]$. Recall that we say that a probability measure $\mu$ on the real line satisfies the transport-entropy inequality $\mathbf{T}_{\min \left\{x^{2},|x|^{\alpha}\right\}}(a)$ if for any probability measure $\sigma$ on the real line

$$
\mathcal{T}_{\alpha, a}(\mu, \sigma) \leq H(\sigma \mid \mu),
$$

where $\mathcal{T}_{\alpha, a}$ is the optimal transport cost between the measures $\mu$ and $\sigma$ with respect to the cost function $t \mapsto \min \left\{(a t)^{2},|a t|^{\alpha}\right\}$, i.e.,

$$
\mathcal{T}_{\alpha, a}(\mu, \sigma)=\inf _{\pi} \int_{\mathbb{R}} \int_{\mathbb{R}} \min \left\{(a(x-y))^{2},|a(x-y)|^{\alpha}\right\} d \pi(x, y),
$$

where the infimum runs over the set of couplings between $\mu$ and $\sigma$, and $H(\sigma \mid \mu)$ stands for the relative entropy of $\sigma$ with respect to $\mu$.

Proposition 4 Let $\alpha \in(1,2]$. The measure $v_{\alpha}$ satisfies the transport-entropy inequality $\mathbf{T}_{\min \left\{x^{2},|x|^{\alpha}\right\}}(a)$ with some constant $a>0$ depending only on $\alpha$.

One can also wonder what happens if we allow the potential $V$ to have even bigger oscillations. For $\alpha>1$ and $\lambda>1$ define

$$
V_{\alpha, \lambda}(x)=|x+\lambda \sin (x)|^{\alpha}, \quad x \in \mathbb{R},
$$

and let $v_{\alpha, \lambda}$ be the probability measure with density proportional to $\exp \left(-V_{\alpha, \lambda}\right)$.

Proposition 5 Let $\alpha>1$ and $\lambda>1$. The measure $v_{\alpha, \lambda}$ does not satisfy the Poincaré inequality (2).

\subsection{Proofs}

In the next two proofs we shall omit the subscript $\alpha$ in the notation and write $V, v$, and $Z$ instead of $V_{\alpha}, v_{\alpha}$ and $Z_{\alpha}$, respectively.

Proof of Proposition 3 Fix $\alpha>1$. Let us start with proving the implication $(i) \Longrightarrow(i i)$, i.e., that the Latała-Oleszkiewicz inequality (5) holds for $r \leq r_{0}(\alpha)$. For $x>0$ we have $V(x)=(x+\sin (x))^{\alpha}$ and

$$
V^{\prime}(x)=\alpha(x+\sin (x))^{\alpha-1}(1+\cos (x)) .
$$

Denote for simplicity $\beta=(\alpha-1) / 3$. For $x>0$ such that $1-\beta x^{-\beta-1} \geq 0$, we have

$$
\begin{aligned}
\int_{x}^{\infty} e^{-V(t)} d t & =\int_{x}^{x+x^{-\beta}} e^{-V(t)} d t+\int_{x+x^{-\beta}}^{\infty} e^{-V(t)} d t \\
& \leq \frac{e^{-V(x)}}{x^{\beta}}+\int_{x}^{\infty} e^{-V\left(u+u^{-\beta}\right)}\left(1-\beta u^{-\beta-1}\right) d u \\
& \leq \frac{e^{-V(x)}}{x^{\beta}}+\int_{x}^{\infty} e^{-V\left(u+u^{-\beta}\right)} d u
\end{aligned}
$$


where we used the fact the $V$ is increasing on $(0, \infty)$ and substituted $t=u+u^{-\beta}$ in the second integral. Note that, by the convexity of the function $x \mapsto x^{\alpha}, x>0$,

$$
\begin{aligned}
V\left(u+u^{-\beta}\right)-V(u) & =\left(u+u^{-\beta}+\sin \left(u+u^{-\beta}\right)\right)^{\alpha}-(u+\sin (u))^{\alpha} \\
& \geq \alpha(u+\sin (u))^{\alpha-1}\left(u^{-\beta}+\sin \left(u+u^{-\beta}\right)-\sin (u)\right) \\
& =\alpha(u+\sin (u))^{\alpha-1}\left(u^{-\beta}+2 \sin \left(u^{-\beta} / 2\right) \cos \left(u+u^{-\beta} / 2\right)\right) \\
& \geq \alpha(u+\sin (u))^{\alpha-1}\left(u^{-\beta}-2 \sin \left(u^{-\beta} / 2\right)\right) \\
& \geq c_{1} u^{\alpha-1} u^{-3 \beta}=c_{1}
\end{aligned}
$$

for some $c_{1}=c_{1}(\alpha)>0$ (for, say, $u \geq 1$ ). Thus Eq. 24 implies that for sufficiently large $x$,

$$
\int_{x}^{\infty} e^{-V(t)} d t \leq \frac{1}{1-e^{-c_{1}}} \cdot \frac{e^{-V(x)}}{x^{(\alpha-1) / 3}} .
$$

We proceed similarly with $\int_{0}^{x} e^{V(t)} d t$. First note that, for $t \geq 2 \pi$,

$$
V(t-2 \pi)+(2 \pi)^{\alpha}=(t-2 \pi+\sin (t))^{\alpha}+(2 \pi)^{\alpha} \leq(t+\sin (t))^{\alpha}=V(t),
$$

since $\alpha \geq 1$. Hence,

$$
\int_{2(k-1) \pi}^{2 k \pi} e^{V(t)} d t=\int_{2 k \pi}^{2(k+1) \pi} e^{V(t-2 \pi)} d t \leq e^{-(2 \pi)^{\alpha}} \int_{2 k \pi}^{2(k+1) \pi} e^{V(t)} d t
$$

and consequently, for $x \geq 2 \pi$,

$$
\begin{aligned}
\int_{0}^{x} e^{V(t)} d t & \leq\left(1+\sum_{k=0}^{\infty} e^{-k(2 \pi)^{\alpha}}\right) \int_{x-2 \pi}^{x} e^{V(t)} d t \\
& =\left(1+\frac{1}{1-e^{-(2 \pi)^{\alpha}}}\right) \int_{x-2 \pi}^{x} e^{V(t)} d t
\end{aligned}
$$

(recall that $V$ is increasing on $(0, \infty)$ ).

As above, denote for simplicity $\beta=(\alpha-1) / 3$. For $x>2 \pi$ we have

$$
\begin{aligned}
\int_{x-2 \pi}^{x} e^{V(t)} d t & =\int_{x-x^{-\beta}}^{x} e^{V(t)} d t+\int_{x-2 \pi}^{x-x^{-\beta}} e^{V(t)} d t \\
& \leq \frac{e^{V(x)}}{x^{\beta}}+\int_{l(x)}^{x} e^{V\left(u-u^{-\beta}\right)}\left(1+\beta u^{-\beta-1}\right) d u, \\
& \leq \frac{e^{V(x)}}{x^{\beta}}+\left(1+\beta(x-2 \pi)^{-\beta-1}\right) \int_{x-2 \pi}^{x} e^{V\left(u-u^{-\beta}\right)} d u,
\end{aligned}
$$

where we used the fact the $V$ is increasing on $(0, \infty)$ and substituted $t=u-u^{-\beta}$ in the second integral $\left(l(x)>x-2 \pi\right.$ is the unique number such that $\left.x-2 \pi=l(x)-l(x)^{-\beta}\right)$. Note that

$$
\begin{aligned}
& V\left(u-u^{-\beta}\right)-V(u)=\left(u-u^{-\beta}+\sin \left(u-u^{-\beta}\right)\right)^{\alpha}-(u+\sin (u))^{\alpha} \\
& \quad \leq \quad-\alpha\left(u-u^{-\beta}+\sin \left(u-u^{-\beta}\right)\right)^{\alpha-1}\left(u^{-\beta}-\sin \left(u-u^{-\beta}\right)+\sin (u)\right) \\
& \quad=\quad-\alpha\left(u-u^{-\beta}+\sin \left(u-u^{-\beta}\right)\right)^{\alpha-1}\left(u^{-\beta}-2 \sin \left(u^{-\beta} / 2\right) \cos \left(u-u^{-\beta} / 2\right)\right) \\
& \quad \leq \quad-\alpha\left(u-u^{-\beta}+\sin \left(u-u^{-\beta}\right)\right)^{\alpha-1}\left(u^{-\beta}-2 \sin \left(u^{-\beta} / 2\right)\right) \\
& \quad \leq \quad-c_{2} u^{\alpha-1} u^{-3 \beta}=-c_{2}
\end{aligned}
$$


for some $c_{2}=c_{2}(\alpha)>0$ and sufficiently large $u>0$. Thus Eq. 26 implies that for sufficiently large $x$,

$$
\begin{gathered}
\int_{x-2 \pi}^{x} e^{V(t)} d t \leq \frac{e^{V(x)}}{x^{\beta}}+ \\
\left(1+\beta(x-2 \pi)^{-\beta-1}\right) e^{-c_{2}} \int_{x-2 \pi}^{x} e^{V(u)} d u \\
\leq \frac{e^{V(x)}}{x^{\beta}}+e^{-\widetilde{c}_{2}} \int_{x-2 \pi}^{x} e^{V(u)} d u,
\end{gathered}
$$

for some $\widetilde{c}_{2}=\widetilde{c}_{2}(\alpha)>0$. Thus, for sufficiently large $x$,

$$
\int_{0}^{x} e^{V(t)} d t \leq \frac{1+\frac{1}{1-e^{-(2 \pi)^{\alpha}}}}{1-e^{-\widetilde{c}_{2}}} \cdot \frac{e^{V(x)}}{x^{(\alpha-1) / 3}} .
$$

For $q>2$ the function $t \mapsto t \log ^{2 / q}(1+1 /(2 t))$ is increasing for small enough positive $t$. Using Eqs. 25 and 27, we see that for sufficiently large $x>0$,

$$
\begin{aligned}
v([x & , \infty]) \log ^{2 / r^{\prime}}\left(1+\frac{1}{2 v([x, \infty))}\right) \int_{0}^{x} e^{V(t)} d t \\
& \lesssim \frac{e^{-V(x)}}{x^{(\alpha-1) / 3}} \log ^{2 / r^{\prime}}\left(Z e^{V(x)} x^{(\alpha-1) / 3}\right) \frac{e^{V(x)}}{x^{(\alpha-1) / 3}} \\
& \lesssim \frac{V(x)^{2 / r^{\prime}}}{x^{2(\alpha-1) / 3}} \lesssim x^{2\left(\alpha / r^{\prime}-(\alpha-1) / 3\right)}
\end{aligned}
$$

(we omit multiplicative constants not depending on $x$ ). Clearly, if $1<r \leq r_{0}(\alpha)$, then this is bounded as $x \rightarrow \infty$, and by the Barthe-Roberto criterion (see Eq. 18 above) the Latała-Oleszkiewicz inequality with parameter $r$ does hold.

The implication $(i i) \Longrightarrow$ (iii) holds by Theorem 2 .

It remains to show that if $v$ satisfies a modified log-Sobolev inequality with parameter $r$ then necessarily $r \leq r_{0}(\alpha)$ (i.e., the implication (iii) $\Longrightarrow(i)$ holds). This can be seen by focusing on the points $x_{k}=(2 k+1) \pi$ where $V^{\prime}$ vanishes and the estimates from the proof of implication $(i) \Longrightarrow(i i)$ can be reversed up to multiplicative constants. Indeed, for $k$ large enough one can find a constant $c_{3}$ such that for $y \in\left[x_{k}-k^{-\beta}, x_{k}+k^{-\beta}\right]$,

$$
\left|V^{\prime}(y)\right| \leq c_{3} x_{k}^{\alpha-1} k^{-2 \beta} .
$$

For $k$ and $y$ as above,

$$
\left|V(y)-V\left(x_{k}\right)\right| \leq c_{3} x_{k}^{\alpha-1} k^{-3 \beta} \leq c_{4},
$$

using here that $\beta=(\alpha-1) / 3$. Thus,

$$
\begin{aligned}
\int_{x_{k}}^{\infty} e^{-V(x)} d x & \geq \int_{x_{k}}^{x_{k}+k^{-\beta}} e^{-V(x)} d x \geq k^{-\beta} e^{-V\left(x_{k}\right)} e^{-c_{4}}, \\
\left(\int_{0}^{x_{k}} e^{(r-1) V(x)} d x\right)^{\frac{1}{r-1}} & \geq\left(\int_{x_{k}-k^{-\beta}}^{x_{k}} e^{(r-1) V(x)} d x\right)^{\frac{1}{r-1}} \geq k^{-\beta /(r-1)} e^{V\left(x_{k}\right)} e^{-c_{4}} .
\end{aligned}
$$


Consequently, as the function $t \mapsto t \log (1 / t)$ is increasing for small enough positive $t$, for $k$ sufficiently large we can write:

$$
\begin{aligned}
v\left(\left[x_{k}, \infty\right)\right) \log \left(\frac{1}{v\left(\left[x_{k}, \infty\right)\right)}\right)\left(\int_{0}^{x_{k}} e^{(r-1) V(t)} d t\right)^{1 /(r-1)} \\
\geq \frac{e^{-V\left(x_{k}\right)}}{k^{\beta}} \log \left(e^{V\left(x_{k}\right)} k^{\beta}\right) \frac{e^{V\left(x_{k}\right)}}{k^{\beta /(r-1)}} \\
\geq \frac{V\left(x_{k}\right)}{k^{\beta r^{\prime}}} \geq \frac{(2 k \pi)^{\alpha}}{k^{\beta r^{\prime}}} .
\end{aligned}
$$

If $v$ satisfies the modified log-Sobolev inequality with parameter $r$, then by the BartheRoberto criterion (see Eq. 19 above) the latter quantity remains bounded from above when $k \rightarrow \infty$ (note that this requires Assumption Eq. 20, which is verified since Eq. 27 is valid for $(r-1) V$ instead of $V$, with different numerical constants). This forces $\alpha \leq \beta r^{\prime}$, or equivalently $r \leq r_{0}(\alpha)$.

Proof of Proposition 4 Fix $\alpha \in(1,2]$ and denote, for $t \geq 0$,

$$
e^{-N(t)}=\frac{2}{Z} \int_{t}^{\infty} e^{-V(s)} d s .
$$

Let $F_{v}$ and $F_{\text {exp }}$ be the cumulative distribution functions of $v$ and the symmetric exponential measure with density $\frac{1}{2} e^{-|x|}$ respectively.

By Proposition 3, v satisfies the Latała-Oleszkiewicz inequality with $r_{0}(\alpha)>1$, so it satisfies the Poincaré inequality. Thus, by Theorem 1.1 of [16], in order to prove the assertion it suffices to show that there exists $b=b(\alpha)>0$ such that

$$
\left|F_{v}^{-1}\left(F_{\exp }(x)\right)-F_{v}^{-1}\left(F_{\exp }(y)\right)\right| \leq \frac{1}{b}(1+|x-y|)^{1 / \alpha}
$$

for all $x, y \in \mathbb{R}$.

Note that for $x \geq 0$ we have $\tilde{x}=F_{v}^{-1}\left(F_{\exp }(x)\right)$ if and only if

$$
1-\frac{1}{2} e^{-N(\tilde{x})}=F_{v}(\tilde{x})=F_{\exp }(x)=1-\frac{1}{2} e^{-x} .
$$

Thus it suffices to check that there exists $b=b(\alpha)>0$ such that

$$
b^{\alpha}|x-y|^{\alpha} \leq 1+|N(|x|) \operatorname{sgn}(x)-N(|y|) \operatorname{sgn}(y)|
$$

for all $x, y \in \mathbb{R}$ (recall that $v$ is symmetric).

If $|x-y| \leq 2 \pi$, then one can guarantee that Eq. 28 holds simply by taking $b \leq(2 \pi)^{-1}$. Let therefore consider the case when $|x-y| \geq 2 \pi$. We have two cases:

1. $x, y$ are of different signs,

2. $x, y$ are of the same sign.

Case 1. In the first case we have

$$
b^{\alpha}|x-y|^{\alpha}=b^{\alpha}(|x|+|y|)^{\alpha} \leq 2^{\alpha-1} b^{\alpha}\left(|x|^{\alpha}+|y|^{\alpha}\right) .
$$


From the proof of Proposition 3 we know that for sufficiently large $t>0$ we have

$$
\frac{1}{2} e^{-N(t)}=\frac{1}{Z} \int_{t}^{\infty} e^{-V(s)} d s \leq \frac{1}{Z\left(1-e^{-c_{1}(\alpha)}\right)} \cdot \frac{e^{-V(t)}}{t^{(\alpha-1) / 3}}
$$

(see Eq. 25). Thus, for sufficiently large $t>0$,

$$
N(t) \geq V(t)=|t+\sin (t)|^{\alpha} \geq \frac{1}{2} t^{\alpha} .
$$

Therefore, we can choose $b>0$ to be such that $2^{\alpha-1} b^{\alpha} t^{\alpha} \leq N(t)+\frac{1}{2}$ holds for all $t>0$. Then

$$
b^{\alpha}|x-y|^{\alpha} \leq 2^{\alpha-1} b^{\alpha}\left(|x|^{\alpha}+|y|^{\alpha}\right) \leq 1+N(|x|)+N(|y|),
$$

which is exactly Eq. 28 in the case when $x, y$ are of different signs.

Case 2. Suppose now that $x, y$ are of the same sign, say $x \geq y+2 \pi \geq y \geq 0$. Observe that, for $t>0$ and $k \in \mathbb{N}$,

$$
V(t+2 k \pi)=|t+\sin (t)+2 k \pi|^{\alpha} \geq V(t)+(2 k \pi)^{\alpha} .
$$

Thus, for $t>0$ and $s \geq 2 \pi$,

$$
V(t+s) \geq V(t+2 \pi\lfloor s /(2 \pi)\rfloor) \geq V(t)+(2 \pi)^{\alpha}\lfloor s /(2 \pi)\rfloor^{\alpha} \geq V(t)+s^{\alpha} / 2^{\alpha} .
$$

Therefore, for $t>0$ and $s \geq 2 \pi$,

$$
\begin{aligned}
e^{-N(t+s)} & =\frac{2}{Z} \int_{t+s}^{\infty} e^{-V(u)} d u=\frac{2}{Z} \int_{t}^{\infty} e^{-V(u+s)} d u \\
& \leq \frac{2}{Z} \int_{t}^{\infty} e^{-V(u)-s^{\alpha} / 2^{\alpha}} d u=e^{-N(t)-s^{\alpha} / 2^{\alpha}}
\end{aligned}
$$

Thus, if we take $b \leq 1 / 2$, then Eq. 28 holds also for $x \geq y+2 \pi \geq y \geq 0$ (we substitute $x=t+s, y=t$ ). This finishes the proof.

Proof of Proposition 5 Fix $\lambda, \alpha>1$. We write $V$ for $V_{\alpha, \lambda}$. For sufficiently large $x>0$ we have

$$
V^{\prime}(x)=\alpha(x+\lambda \sin (x))^{\alpha-1}(1+\lambda \cos (x)) .
$$

Denote $x_{k}=(2 k+1) \pi, k \in \mathbb{N}$. There exists $\delta_{0}>0$ such that $V$ is decreasing on $\left[x_{k}-\right.$ $\left.\delta_{0}, x_{k}+\delta_{0}\right]$ for sufficiently large $k$. Thus

$$
\int_{x_{k}}^{\infty} e^{-V(t)} d t \geq \int_{x_{k}}^{x_{k}+\delta_{0}} e^{-V(t)} d t \geq \delta_{0} e^{-V\left(x_{k}\right)}
$$

Moreover, by the convexity of the function $x \mapsto x^{\alpha}(x>0)$, for $h \in\left[0, \delta_{0}\right]$ we have

$$
\begin{aligned}
V\left(x_{k}-h\right)-V\left(x_{k}\right) & =\left(x_{k}-h+\lambda \sin \left(x_{k}-h\right)\right)^{\alpha}-\left(x_{k}+\lambda \sin \left(x_{k}\right)\right)^{\alpha} \\
& \geq \alpha\left(x_{k}+\lambda \sin \left(x_{k}\right)\right)^{\alpha-1}\left(-h+\lambda \sin \left(x_{k}-h\right)\right) \\
& =\alpha x_{k}^{\alpha-1}(\lambda \sin (h)-h) .
\end{aligned}
$$


Hence

$$
\begin{aligned}
\int_{0}^{x_{k}} e^{V(t)} d t & \geq \int_{x_{k}-\delta_{0}}^{x_{k}} e^{V(t)} d t=\int_{0}^{\delta_{0}} e^{V\left(x_{k}-h\right)} d h \\
& \geq \int_{0}^{\delta_{0}} e^{V\left(x_{k}\right)+\alpha x_{k}^{\alpha-1}(\lambda \sin (h)-h)} d h .
\end{aligned}
$$

Putting together Eqs. 29 and 30, we observe that

$$
\int_{x_{k}}^{\infty} e^{-V(t)} d t \times \int_{0}^{x_{k}} e^{V(t)} d t \geq \delta_{0} \int_{0}^{\delta_{0}} e^{\alpha x_{k}^{\alpha-1}(\lambda \sin (h)-h)} d h
$$

tends to infinity when $k \rightarrow \infty$, since $\lambda \sin (h)-h>0$ for $h$ positive and small enough. By the Muckenhoupt criterion (see Eq. 16), we may conclude that $v$ cannot satisfy any Poincaré inequality.

\subsection{Remarks on a General Setting}

In this final section, we show informally how the method used in the calculations of the previous section can be extended to more general families of measures. The main issue is to derive estimates of the quantities $\int_{x_{0}}^{x} e^{V}$ and $\int_{x}^{\infty} e^{-V}$. For shortness we do not treat separately upper and lower bounds.

The classical approach is based on writing $\int e^{V}=\int \frac{1}{V^{\prime}} \times V^{\prime} e^{V}$ and on an integration by parts. It works if there exist $x_{0}$ and $\varepsilon>0$ such that for $x \geq x_{0}, V$ is of class $\mathcal{C}^{2}, V^{\prime}>0$ and $\left|\frac{V^{\prime \prime}}{\left(V^{\prime}\right)^{2}}\right| \leq 1-\varepsilon$. In this case, up to multiplicative constants which depend on $\varepsilon$, for $x \geq x_{0}$,

$$
\int_{x}^{\infty} e^{-V} \approx \frac{e^{-V(x)}}{V^{\prime}(x)}, \quad \int_{x_{0}}^{x} e^{V} \approx \frac{e^{V(x)}}{V^{\prime}(x)} .
$$

This approach cannot work if $V^{\prime}$ vanishes for arbitrarily large values, as it was the case for $V_{\alpha}$. The approach that we used for $v_{\alpha}$ can still be applied in such situations, when the potential is, in some sense, essentially increasing. The key parameter at point $x$ is a number $\theta(x)>0$ so that for some constants $C \geq c>0$ (independent of $x$ ),

$$
\begin{gathered}
\forall y \in[x-\theta(x), x+\theta(x)], \quad|V(y)-V(x)| \leq C, \\
V(x+\theta(x)) \geq V(x)+c, \\
V(x-\theta(x)) \leq V(x)+c .
\end{gathered}
$$

In words, $V$ is essentially constant on $[x-\theta(x), x+\theta(x)]$, but does increase between the left endpoint and the center, and between the center and the right endpoint. For the upper bound, one also needs $V$ to grow at least linearly: $V(x+K) \geq V(x)+c$. Under some additional assumptions (e.g., $\theta^{\prime}$ is small enough compared to $c$ ), one gets

$$
\int_{x}^{\infty} e^{-V} \approx \theta(x) e^{-V(x)}, \quad \int_{x_{0}}^{x} e^{V} \approx \theta(x) e^{V(x)} .
$$

Note that when $V^{\prime}(x)>0,1 / V^{\prime}(x)$ is heuristically the scale at which $V$ moves by 1 , which makes a connection with the classical approach. Let us also mention that for measures having the latter properties, the Latała-Oleszkiewicz and the modified log-Sobolev inequality 
with parameters $r$ will be true simultaneously. Indeed if $\theta$ is bounded from above, then Condition (20) should be verified. Then the quantities $B_{L O(r)}^{+}$and $B_{m L S(r)}^{+}$are comparable since for $x$ large

$$
\begin{aligned}
& \left(\int_{x}^{\infty} e^{-V}\right) \log ^{2 / r^{\prime}}\left(1+\frac{1}{\int_{x}^{\infty} e^{-V}}\right) \int_{0}^{x} e^{V(t)} d t \approx \theta(x)^{2}(V(x)+\log \theta(x))^{2 / r^{\prime}}, \\
& \left(\int_{x}^{\infty} e^{-V}\right) \log \left(\frac{1}{\int_{x}^{\infty} e^{-V}}\right)\left(\int_{0}^{x} e^{(r-1) V(t)} d t\right)^{1 /(r-1)} \approx \theta(x)^{r^{\prime}}(V(x)+\log \theta(x)) .
\end{aligned}
$$

Let us conclude with a simple observation about potentials which are nonincreasing on infinitely many intervals (variants involving essentially nonincreasing ones can be written).

Lemma 3 Let $\mu$ be a probability measure on the real line with density proportional to $\exp (-V(x))$ for some locally bounded $V: \mathbb{R} \rightarrow \mathbb{R}$. Suppose that there exists $\varepsilon>0$ and a sequence of positive real numbers $x_{n} \rightarrow \infty$, such that $V$ is nonincreasing on $\left(x_{n}-\varepsilon, x_{n}+\varepsilon\right)$. Then $\mu$ does not satisfy the Latała-Oleszkiewicz inequality (5) with any parameter $r \in$ $(1,2)$.

Proof From the assumption about the monotonicity of $V$ on the intervals $\left(x_{n}-\varepsilon, x_{n}+\varepsilon\right)$, we get

$$
\int_{x_{n}}^{\infty} e^{-V(t)} d t \int_{0}^{x_{n}} e^{V(t)} d t \geq \varepsilon e^{-V\left(x_{n}\right)} \cdot \varepsilon e^{V\left(x_{n}\right)}=\varepsilon^{2} .
$$

Moreover, $\log ^{2 / r^{\prime}}\left(1+\frac{1}{2 \mu([x, \infty))}\right) \rightarrow+\infty$ for any $r \in(1,2)$. Thus, by the Barthe-Roberto criterion (see Eq. 18 above) the Latała-Oleszkiewicz inequality (5) cannot hold, with any parameter $r \in(1,2)$.

The above result should be compared to Proposition 5. Observe that measures satisfying the hypotheses of the Lemma may verify a Poincaré inequality. This is the case for the potential $V(x)=\lfloor|x|\rfloor$ involving the integer part. This potential is constant on every interval $[k, k+1), \in \mathbb{N}$. Nevertheless $V(x)$ is a bounded additive perturbation of the potential $|x|$ of the symmetric exponential distribution, hence the associated measure satisfies a Poincaré inequality.

Open Access This article is licensed under a Creative Commons Attribution 4.0 International License, which permits use, sharing, adaptation, distribution and reproduction in any medium or format, as long as you give appropriate credit to the original author(s) and the source, provide a link to the Creative Commons licence, and indicate if changes were made. The images or other third party material in this article are included in the article's Creative Commons licence, unless indicated otherwise in a credit line to the material. If material is not included in the article's Creative Commons licence and your intended use is not permitted by statutory regulation or exceeds the permitted use, you will need to obtain permission directly from the copyright holder. To view a copy of this licence, visit http://creativecommonshorg/licenses/by/4.0/. 


\section{References}

1. Adamczak, R., Strzelecki, M.: Modified log-Sobolev inequalities for convex functions on the real line. Sufficient conditions. Studia Math. 230(1), 59-93 (2015). https://doi.org/10.4064/sm8319-12-2015

2. Ané, C., Blachère, S., Chafaï, D., Fougères, P., Gentil, I., Malrieu, F., Roberto, C., Scheffer, G.: Sur les inégalités de Sobolev logarithmiques. Panoramas et Synthèses (Panoramas and Syntheses), vol. 10. Société Mathématique de France, Paris (2000). With a preface by Dominique Bakry and Michel Ledoux

3. Barthe, F., Kolesnikov, A.V.: Mass transport and variants of the logarithmic Sobolev inequality. J. Geom. Anal. 18(4), 921-979 (2008). https://doi.org/10.1007/s12220-008-9039-6

4. Barthe, F., Roberto, C.: Sobolev inequalities for probability measures on the real line. Studia Math. 159(3), 481-497 (2003). https://doi.org/10.4064/sm159-3-9. Dedicated to Professor Aleksander Pełczyński on the occasion of his 70th birthday (Polish)

5. Barthe, F., Roberto, C.: Modified logarithmic Sobolev inequalities on $\mathbb{R}$. Potential Anal. 29(2), 167-193 (2008). https://doi.org/10.1007/s11118-008-9093-5

6. Barthe, F., Cattiaux, P., Roberto, C.: Interpolated inequalities between exponential and Gaussian, Orlicz hypercontractivity and isoperimetry. Rev. Mat. Iberoam. 22(3), 993-1067 (2006). https://doi.org/ 10.4171/RMI/482

7. Beckner, W.: A generalized Poincaré inequality for Gaussian measures. Proc. Am. Math. Soc. 105(2), 397-400 (1989). https://doi.org/10.2307/2046956

8. Bobkov, S., Götze, F.: Exponential integrability and transportation cost related to logarithmic Sobolev inequalities. J. Funct. Anal. 163(1), 1-28 (1999). https://doi.org/10.1006/jfan.1998.3326

9. Bobkov, S., Ledoux, M.: Poincaré's inequalities and Talagrand's concentration phenomenon for the exponential distribution. Probab. Theory Relat. Fields 107(3), 383-400 (1997). https://doi.org/10.1007/ s004400050090

10. Cattiaux, P.: Hypercontractivity for perturbed diffusion semigroups. Ann. Fac. Sci. Toulouse Math. (6) 14(4), 609-628 (2005). https://doi.org/10.5802/afst.1105

11. Cattiaux, P., Guillin, A.: On quadratic transportation cost inequalities. J. Math. Pures Appl. (9) 86(4), 341-361 (2006). https://doi.org/10.1016/j.matpur.2006.06.003

12. Cattiaux, P., Guillin, A., Wu, L.M.: Some remarks on weighted logarithmic Sobolev inequality. Indiana Univ. Math. J. 60(6), 1885-1904 (2011). https://doi.org/10.1512/iumj.2011.60.4405

13. Gentil, I., Guillin, A., Miclo, L.: Modified logarithmic Sobolev inequalities and transportation inequalities. Probab. Theory Relat. Fields 133(3), 409-436 (2005). https://doi.org/10.1007/s00440-005-0432-9

14. Gozlan, N.: A characterization of dimension free concentration in terms of transportation inequalities. Ann. Probab. 37(6), 2480-2498 (2009). https://doi.org/10.1214/09-AOP470

15. Gozlan, N.: Poincaré inequalities and dimension free concentration of measure. Ann. Inst. Henri Poincaré Probab. Stat. 46(3), 708-739 (2010). https://doi.org/10.1214/09-AIHP209

16. Gozlan, N.: Transport-entropy inequalities on the line. Electron. J. Probab. 17(49), 1-18 (2012). https://doi.org/10.1214/EJP.v17-1864

17. Gozlan, N., Roberto, C., Samson, P.M.: From dimension free concentration to the Poincaré inequality. Calc. Var. Partial Differ. Equ. 52(3-4), 899-925 (2015). https://doi.org/10.1007/s00526-014-0737-6

18. Latała, R., Oleszkiewicz, K.: Between Sobolev and Poincaré. In: Geometric aspects of functional analysis, Lecture Notes in Math, vol. 1745, pp. 147-168. Springer, Berlin (2000). https://doi.org/10. 1007/BFb0107213

19. Ledoux, M.: The concentration of measure phenomenon. Mathematical Surveys and Monographs, vol. 89. American Mathematical Society, Providence (2001)

20. Miclo, L.: Quand est-ce que des bornes de Hardy permettent de calculer une constante de Poincaré exacte sur la droite. Ann. Fac. Sci. Toulouse Math. (6) 17(1), 121-192 (2008). https://doi.org/10.5802/afst.1179

21. Rockafellar, R.T.: Convex analysis. Princeton Mathematical Series, vol. 28. Princeton University Press, Princeton (1970)

22. Shao, J.: Modified logarithmic Sobolev inequalities and transportation cost inequalities in $\mathbb{R}^{n}$. Potential Anal. 31(2), 183-202 (2009). https://doi.org/10.1007/s11118-009-9131-y

23. Talagrand, M.: A new isoperimetric inequality and the concentration of measure phenomenon. In: Geometric aspects of functional analysis (1989-90), Lecture Notes in Math., vol. 1469, pp. 94-124. Springer, Berlin (1991). https://doi.org/10.1007/BFb0089217

24. Talagrand, M.: Concentration of measure and isoperimetric inequalities in product spaces. Inst. Hautes Études Sci. Publ. Math. (81), 73-205 (1995). https://doi.org/10.1007/BF02699376

25. Talagrand, M.: Transportation cost for Gaussian and other product measures. Geom. Funct. Anal. 6(3), 587-600 (1996). https://doi.org/10.1007/BF02249265 
26. Wang, F.Y.: A generalization of Poincaré and log-Sobolev inequalities. Potential Anal. 22(1), 1-15 (2005). https://doi.org/10.1007/s11118-004-4006-8

27. Wang, F.Y.: From super Poincaré to weighted log-Sobolev and entropy-cost inequalities. J. Math. Pures Appl. (9) 90(3), 270-285 (2008). https://doi.org/10.1016/j.matpur.2008.06.004

Publisher's Note Springer Nature remains neutral with regard to jurisdictional claims in published maps and institutional affiliations. 